\title{
Error of the network approximation for densely packed composites with irregular geometry
}

\author{
Leonid Berlyand $^{*} \quad$ Alexei Novikov ${ }^{\dagger}$
}

August 12, 2001

\section{Contents}

1 Introduction. Overview of the paper 2

2 Formulation 7

2.1 The mathematical model . . . . . . . . . . . . . . 7

2.2 Direct and dual variational formulations . . . . . . . . . . . . 9

3 Construction of the discrete network $\quad 10$

3.1 Effective conductivity for periodic square and hexagonal lattices . . . 10

3.2 The discrete network for densely packed high contrast composites . . 15

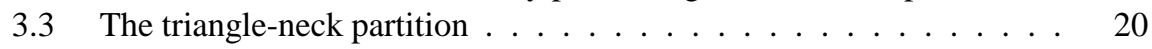

3.4 The discrete minimization problem . . . . . . . . . . . 24

4 Variational bounds on the effective conductivity 29

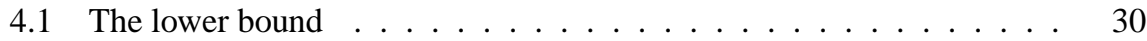

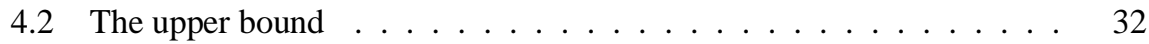

4.3 The error estimate . . . . . . . . . . . . . . . . . 34

4.4 A numerical illustration . . . . . . . . . . . . . . 42

5 Conclusions $\quad 45$

A Appendix. Auxiliary estimates 46

A.1 Estimates on the relative neck widths . . . . . . . . . . . . . . 46

A.2 Gradient estimates inside the necks . . . . . . . . . . . . . . 49

A.3 Comparison of fluxes for the two discrete networks . . . . . . . 51

A.4 Gradient estimates inside the triangles . . . . . . . . . . 53

${ }^{*}$ Department of Mathematics, 414 McAllister Building, Pennsylvania State University, University Park, PA 16802, USA (berlyand@math.psu.edu)

${ }^{\dagger}$ IMA, University of Minnesota, 400 Lind Hall, 207 Church S.E., Minneapolis, MN 55455, USA. Present address: California Institute of Technology, Applied \& Computational Mathematics, 1200 E. California Boulevard, MC 217-50, Pasadena, CA 91125, USA (novikov@acm. caltech.edu) 


\begin{abstract}
We apply a discrete network approximation to the problem of the effective conductivity of the high contrast, highly packed composites. The inclusions are irregularly (randomly) distributed in the hosting medium, so that a significant fraction of them may not participate in the conducting spanning cluster. For this class of inclusion distributions we derive a discrete network approximation and obtain an a priori error estimate for this approximation in which all the constants are explicitly computed. Explicit dependence on the irregular geometry of the inclusions' array is obtained.

We use variational techniques to provide rigorous mathematical justification for the approximation and its error estimate.
\end{abstract}

Key words. effective conductivity, discrete network, error estimate, variational bounds

AMS subject classifications. 74Q05, 35Q72, 94C05

\title{
1 Introduction. Overview of the paper
}

We study the effective properties such as the effective conductivity or the effective dielectric constant of composite materials in which a large number of inclusions (filler) are irregularly (randomly) distributed in a homogeneous hosting medium (matrix). For ease of presentation and clarity we concentrate here on the effective conductivity. We are particularly interested in the case of the high contrast, highly packed particular composites, that is when the conductivity of the inclusions is much larger than the conductivity of the hosting medium and the volume fraction of the inclusions is very high. High contrast composites are extremely attractive for the design of new materials with physical properties better than those of their constituents. The case when the concentration of the filling inclusions is high is particularly relevant to polymer/ceramic composites, because a polymer matrix compensates for the brittle nature of ceramics which is their main weakness. A survey on the relevant engineering problems in two and three dimensions (fibers and particles in a matrix) can be found in [4].

Our main tool is the discrete network approximation of [4] for a two dimensional composite, where the inclusions are modeled as identical disks. We focus on the two key issues arising for this approximation. The first is the explicit error estimate of the discrete network approximation to the continuum problem of effective conductivity. The second is a quantitative estimate on how the connectivity patterns for various irregular distributions of the inclusions affect the effective conductivity.

The main advantage of our discrete network approximation is that it is easy to implement numerically and at the same time it captures geometric patterns of the location of inclusions in the matrix. The importance of the geometric patterns in evaluation of the effective properties of high contrast composites can be seen in the analysis of periodic structures. It was observed that for such periodic composites of moderate volume fraction, that is away from the almost touching situation, the effective conductivity is of order of the conductivity of the matrix (see, for example, [2], [17], [18], and references therein). In other words, the filler has almost no effect on the effective conductivity. However, in the case of almost touching inclusions, the effective conductivities of two 
periodic structures with different locations of inclusions in the matrix can be significantly different for the same volume fraction. For example, if the contrast ratio of the constituents is assumed to be $\infty$, then for the same volume fraction of disks (equal to $\pi / 4$ ) for the hexagonal lattice, the effective conductivity $\widehat{a}=O(1)$ (see theorem 3.1 in this paper), while for the square lattice $\widehat{a}=\infty$ (see [14]).

The case of irregularly distributed inclusions is less well-understood. Since the volume fraction of the inclusions is high, the irregular connectivity patterns in the whole composite (percolation effects) determine the behavior of the effective properties. Moreover, it was observed that the irregular connectivity patterns of conducting inclusions can greatly increase the effective conductivity. Therefore, there is a need for a simple model that is still able to capture percolation effects. Also, while for a given periodic structure the volume fraction of the inclusions uniquely determines the distances between the inclusions, this is no longer true for irregular structures and one should search for a model with a new parameter, which describes the local geometry when the inclusions are close to touching. Such a model (the network approximation) was proposed in [4] in two dimensions. Based on the Voronoi tessellation the notion of the interparticle distance parameter for closely packed ("randomized" hexagonal) patterns was introduced there. In the present work we generalize this notion for a broad class of geometrical patterns. This is important, because in spite of the fact that the hexagonal lattice is the densest packing in two dimensions, it is hard at present to manufacture particulate composites with this packing of inclusions. Our new approach allows to derive an explicit error estimate for the network approximation. Such estimates are rare in homogenization theory, most of the existing estimates provide an order of the magnitude of the error only. The class of geometrical patterns that can be handled by our approach includes the situation of a nonuniform irregular distribution, when a significant fraction of the inclusions does not participate in the conducting spanning cluster. This approach allows to relax the close packing condition of [4], so that not all the "neighboring" disks are closely spaced. More specifically we introduce and study the $\delta-\mathcal{N}$ close packing condition, which loosely speaking allows for "holes" with the perimeter of order $\mathcal{N} R$ in the conducting spanning cluster. Here $R$ is the radius of the particles, and $\mathcal{N}$ is the "size of holes in the conducting clusters" (see figure 1.1). Thus we account quantitatively for the presence of these holes in the composite.

The question of error estimates was raised by I. Babuska, because the analysis of [4] is asymptotic in the interparticle distance parameter and does not provide an error estimate. The analysis in the present paper does not use asymptotics and it holds for any (small) finite value of the relative interparticle distance parameter. This enables us to prove the following error estimate for the effective conductivity $\widehat{a}$.

$$
\frac{|\widehat{a}-I|}{I} \leq C(\mathcal{N}) \sqrt{\frac{\delta}{R}},
$$

where $I$ is the value of effective conductivity provided by the network approximation, $\delta / R$ is the relative interparticle distance. We evaluate the constant $C(\mathcal{N})$ explicitly. For small perturbations of the hexagonal lattice $\mathcal{N}=3, C(3)=8.68$; for a less dense "almost square" packing $\mathcal{N}=4, C(4)=32.74$; for an arbitrary $\mathcal{N}$ we provide an 


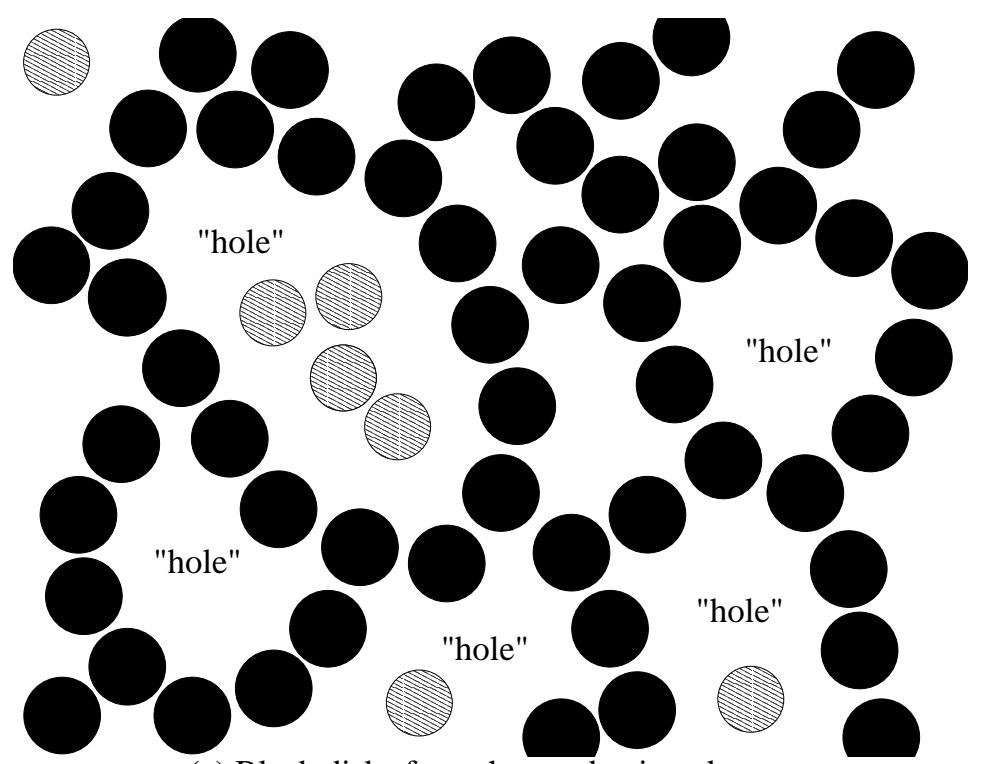

(a) Black disks form the conducting cluster.

Hatched disks do not participate in the cluster.

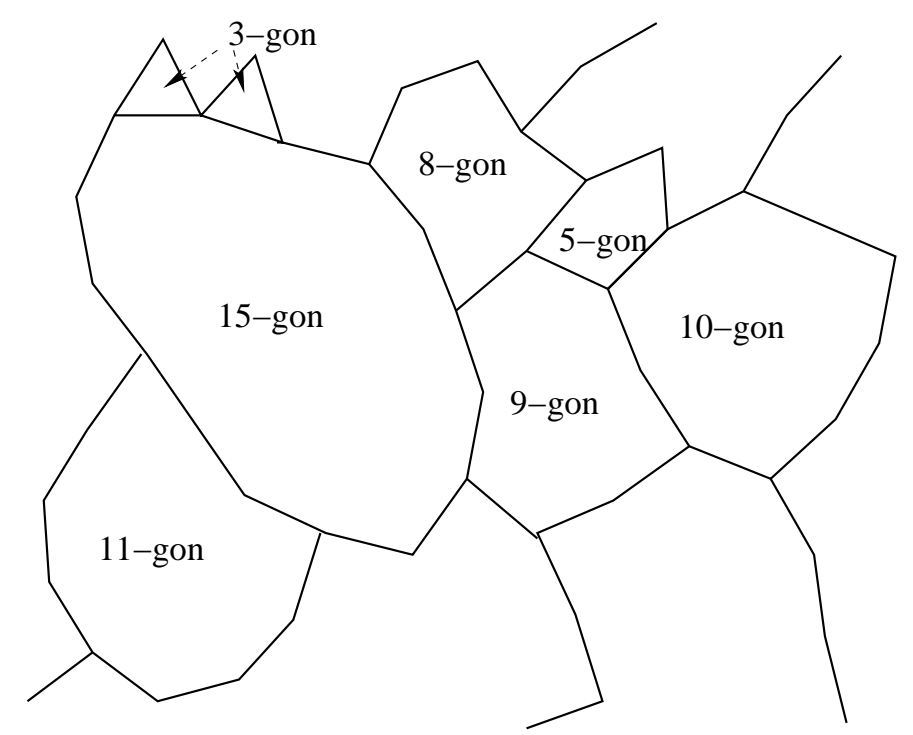

(b) The graph that corresponds to the conducting cluster in (a). An $\mathcal{N}$-gon $\mathcal{N} \geq 4$ corresponds to a "hole" of size $\mathcal{N}$.

Figure 1.1: The conducting cluster in a composite with "holes". 
upper bound $C(\mathcal{N})=9.82 \mathcal{N}^{4}$.

The discrete network models for various high contrast composites have been used extensively in the physics literature (see [1], [11], [13], [15], [20], [21]); however, the relation between the network and the underlying continuum problem was not studied there. In [16], high contrast conductivity problems were first formulated and analyzed using variational methods. There the high contrast field was of the form

$$
e^{S(x) / \epsilon}
$$

with a smooth function $S(x)$. In particular, the asymptotic analysis in the high contrast ratio parameter $\epsilon$ has been carried out in [16] for a random checkerboard model.

For the Kozlov's function (1.2) a network asymptotic approximation in the high contrast parameter $\epsilon$ was developed in [6], [7], [8], [9]. It was rigorously proved in [8] that the network approximates the original continuum problem. The analysis of [8] was carried out for high contrast continuum problems arising in imaging, when the materials' properties are not known and it is convenient to model the high contrast in a simple geometric manner by using (1.2). In this model the key parameter, which determines the conductivity of the edges in the network, is $\sqrt{k_{+} / k_{-}}$, where $k_{+}$and $k_{-}$are the principal curvatures at the saddle points of $S(x)$.

Our analysis applies to a class of physical problems where $S(x)$ is not smooth. In our case $S(x)$ is the characteristic function of the disks

$$
S(x)= \begin{cases}1 & \text { if } x \in \cup D_{i} \\ 0 & \text { if } \in Q_{p}=\Pi-\cup D_{i},\end{cases}
$$

here $D_{i}, i=1, \ldots, N$ are non-overlapping disks, $\Pi=[-L, L] \times[0,1]$ is a a twodimensional rectangular domain, $Q_{p}=\Pi-\cup D_{i}$ is the hosting matrix (see figure 2.1).

Furthermore, in our case the high contrast parameter $\epsilon=0$. In other words, we consider the infinite contrast material with ideally conducting inclusions. This assumption is valid for a variety of particulate composites (particles or fibers in a matrix) and it is in agreement with bounds [10] which imply that if the contrast ratio is greater than several hundreds, then for practical purposes it can be taken to be infinite. More precisely, if one plots the effective conductivity versus the contrast ratio using formulas from [10], then the curve becomes almost horizontal after the contrast ratio reaches values about $10^{3}$.

Our construction of the discrete network approximation for the problem of the effective conductivity is as follows. Suppose the potential $\phi\left(x_{1}, x_{2}\right)$ is a piece-wise differentiable function on $\Pi$, which is constant on each disk $D_{i}$, and takes values -1 and 1 on the lower and the upper boundaries respectively, that is

$$
\phi\left(x_{1}, x_{2}\right)=t_{i}, \text { if }\left(x_{1}, x_{2}\right) \in D_{i}, \phi\left(x_{1},-1\right)=-1, \phi\left(x_{1}, 1\right)=1 .
$$

The goal here is to find the effective conductivity

$$
\widehat{a}=\frac{1}{4 L} \min _{\phi} \int_{Q_{p}}|\nabla \phi|^{2} d \mathbf{x}
$$

where minimum is taken over an appropriate class of admissible functions. 
The discrete network model is based on the observation that the fluxes $\nabla \phi$ are significant only in certain areas ("necks") between closely spaced disks, and in these necks the fluxes can be easily computed. For two disks $D_{i}$ and $D_{j}$ the computation of the flux in the neck $\Pi_{i j}$ between them relies on the observation due to J.B. Keller in [14] that as the distance between the disks $\delta_{i j} \rightarrow 0$, the potential $\phi$ in the neck can be approximated by a linear interpolation between the potentials $t_{i}$ and $t_{j}$ on disks $D_{i}$ and $D_{j}$ respectively.

The discrete network model is a graph where the vertices are the centers of the disks $D_{i}$ and the edges correspond to the necks $\Pi_{i j}$ between neighbors with length $\delta_{i j}$. Neighbors are defined as disks that share a common edge of the Voronoi tessellation of the rectangular domain $\Pi$ with respect to the centers of the disks. To each edge a number (specific flux) $g_{i j}$ is assigned, computed by solving the two-disk problem with fixed potentials $\phi\left(D_{i}\right)=1$ and $\phi\left(D_{j}\right)=0$ in infinite space. The value of effective conductivity, provided by this model is the energy of the discrete network:

$$
I=\frac{1}{4 L} \min _{t_{i}} \sum_{\Pi_{i j}} g_{i j}\left(t_{i}-t_{j}\right)^{2} .
$$

where

$$
g_{i j}=\pi \sqrt{\frac{R}{\delta_{i j}}} .
$$

The number of unknowns in this discrete network approximation is the number of interior disks. All the information about the composite, such as the sizes of the disks and the distances between them, is incorporated in $g_{i j}$. Hence the functional minimization problem (1.4) can be approximated by the simple algebraic minimization problem (1.5), provided we can control the error of this approximation. Now suppose the distances between the necks satisfy the close packing condition

$$
\max \delta_{i j}<\delta \text {. }
$$

Using (1.6) it was shown in [4] that under the close packing condition (1.7) the energy $I$ of the discrete network behaves as

$$
I=O\left(\sqrt{\frac{R}{\delta}}\right), \text { and }|\widehat{a}-I|=O(1), \text { as } \delta \rightarrow 0
$$

and the radius of the disks $R$ is fixed. This result shows that the discrete network is an asymptotically exact approximation to the continuous problem if all the distances between the neighbors are uniformly bounded by $\delta \rightarrow 0$.

Our analysis allows to relax the close packing condition (1.7) and give the error estimates for the fixed relative interparticle distance parameter $\delta / R$. The analysis is based on a new discrete network approximation

$$
I_{0}=\frac{1}{4 L} \min _{t_{i}} \sum_{\Pi_{i j}} g_{i j}^{0}\left(t_{i}-t_{j}\right)^{2} .
$$

The difference between (1.5) and (1.9) is in the choice of coefficients $g_{i j}^{0}$. This choice for (1.9) is entirely geometric which does not require formula (1.6) and can be carried 
out for any distribution of $\delta_{i j}$. Our choice is based on the unique neck-triangle partition of the domain $Q_{p}$ into triangles and necks between disks. For this new discrete network approximation $I_{0}$ we find better error constants $C(\mathcal{N})$ for (1.1). In particular, for small perturbations of the hexagonal lattice $\mathcal{N}=3, C(3)=3.84$; for the almost square packing $\mathcal{N}=4, C(4)=12.73$, for an arbitrary $\mathcal{N}$ we provide an upper bound $C(\mathcal{N})=2.56 \mathcal{N}^{4}$.

The paper is organized as follows. In section 2 we give the formulation of the problem and review techniques of calculus of variations. In section 3 we start with the quantitative comparison of the effective conductivity in two periodic cases: the square lattice and the hexagonal lattice. Then we proceed with the construction of the discrete network approximation. In section 4 we give the main result: the error bounds, based on variational upper and lower estimates. Some technical details of computations for the error estimate are put into appendix A.

\section{Acknowledgments.}

We are grateful to Ivo Babuska for raising the question of the error estimate. The work of L. Berlyand was supported by NSF grant DMS-9971999. Part of this work was done while both authors were visiting members of MSRI, Berkeley. We are grateful for the hospitality and for the support received there.

\section{Formulation}

\subsection{The mathematical model}

Consider a two-dimensional rectangular two-phase composite that consists of a matrix filled by a large number of inclusions. The inclusions are ideally conducting. Assume that all the inclusions are identical non-overlapping disks. The centers of the disks are irregularly distributed in the rectangular domain. The distribution of the disks is dense, that is the characteristic distance between two neighbors is much smaller compared to the radius of the disks.

The mathematical formulation of the problem of effective conductivity comes from a variational minimization problem as follows. Denote the domain occupied by the composite by $\Pi=[-L, L] \times[-1,1]$ (figure 2.1). Denote the disks, that model the inclusions by $D_{i}, i=1, \ldots, N, N$ is the total number of the disks. Then

$$
Q_{p}=\Pi \backslash \cup_{i=1}^{N} D_{i}
$$

is the matrix. Suppose the potential $\phi(x, y)=\phi(\mathbf{x}), \mathbf{x}=(x, y)$ is a piece-wise differentiable function. Apply the potential \pm 1 to the boundaries $y= \pm 1$ respectively:

$$
\phi(\mathbf{x})= \pm 1, \text { on } y= \pm 1 .
$$

Let

$$
V_{p}=\left\{\phi \in H^{1}\left(Q_{p}\right): \phi(\mathbf{x})=t_{i} \text { on } D_{i}, \phi(x, \pm 1)= \pm 1\right\}
$$

Let

$$
I(\phi)=\frac{1}{4 L} \int_{Q_{p}}|\nabla \phi|^{2} d \mathbf{x}, \phi \in V_{p}
$$




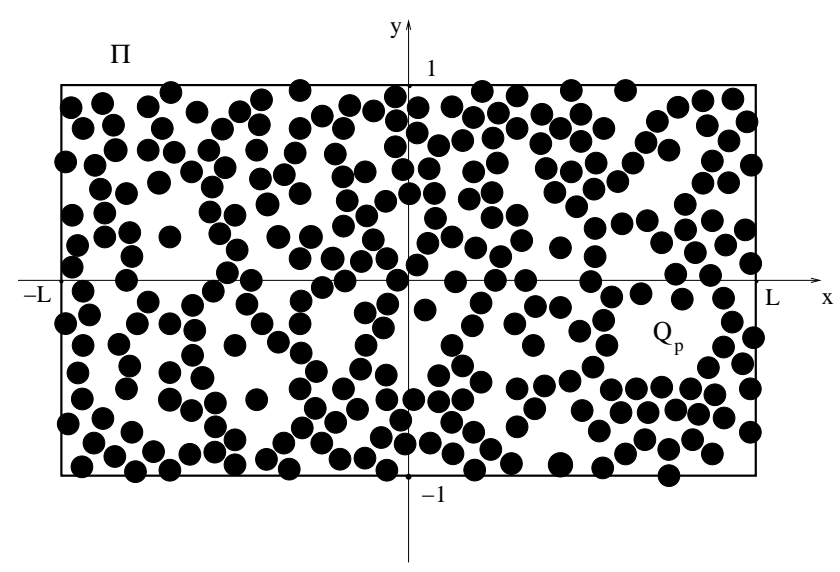

Figure 2.1: The composite

Suppose $\phi$ is the minimizer of the variational problem

$$
\min _{\tilde{\phi} \in V_{p}} I(\tilde{\phi})=I(\phi)
$$

Then $\phi$ is the potential inside the matrix $Q_{p}$. The potential $\phi$ satisfies

$$
\begin{aligned}
& \text { (a) } \Delta \phi=0 \text { in } Q_{p} \\
& \text { (b) } \frac{\partial \phi( \pm L, y)}{\partial \mathbf{n}}=0 \\
& \text { (c) } \int_{\partial D_{i}} \partial \phi / \partial \mathbf{n} d \mathbf{x}=0 \text { for all i, } \\
& \text { (d) } \phi(\mathbf{x})=t_{i} \text { in } \partial D_{i} \\
& \text { (e) } \phi(x, \pm 1)= \pm 1 .
\end{aligned}
$$

Equations (2.5,a-c ) are the Euler-Lagrange equations for the minimization problem (2.4). We apply the potential \pm 1 to the boundaries $y= \pm 1$ respectively ( $2.5 \mathrm{e}$ ) and assume insulating boundary conditions on the vertical boundaries $(2.5 \mathrm{~b})$. The assumption that the disks are ideally conducting implies $(2.5 \mathrm{c}-\mathrm{d})$, where the constants $t_{i}$ in ( $2.5 \mathrm{~d})$ are arbitrary and they should be determined by solving the system (2.5). The integral condition $(2.5 \mathrm{c})$ means that the total flux through any disk is zero. This condition is obtained by integration by parts of the Euler-Lagrange equation and by taking into account the condition that $\phi$ is constant on each $\partial D_{i}$.

The effective conductivity is defined as the total flux per unit length through a horizontal cross-section of the domain. Using $(2.5$ a) in the integration by parts of 
$\int_{Q_{p}} \Delta \phi d \mathbf{x}$ we have that the total fluxes through the horizontal boundaries $y= \pm 1$ are equal

$$
\int_{y=1} \partial \phi / \partial \mathbf{n} d \mathbf{x}=\int_{y=1} \partial \phi / \partial y d \mathbf{x}=\int_{y=-1} \partial \phi / \partial y d \mathbf{x}=-\int_{y=-1} \partial \phi / \partial \mathbf{n} d \mathbf{x}
$$

Therefore for a definition of the effective conductivity we can take for example the flux through the upper boundary $y=1$.

Definition 1 The total flux through the boundary $y=1$ per unit length defined by

$$
\widehat{a}=\frac{1}{2 L} \int_{y=1} \partial \phi / \partial \boldsymbol{n} d \boldsymbol{x}=\frac{1}{2 L} \int_{y=1} \partial \phi / \partial y d \boldsymbol{x} .
$$

is called the effective conductivity.

\subsection{Direct and dual variational formulations}

An equivalent variational formulation of the effective conductivity is given in terms of the Dirichlet's integral $I(\tilde{\phi})$ (equation (2.3)). Suppose $\phi$ is the minimizer of $I(\tilde{\phi})$. By definition

$$
\widehat{a}=\frac{1}{2 L} \int_{y=1} \partial \phi / \partial \mathbf{n} d \mathbf{x}=\frac{1}{2 L} \int_{y=1} \partial \phi / \partial y d \mathbf{x}
$$

then

$$
\widehat{a}=\min _{\tilde{\phi} \in V_{p}} I(\tilde{\phi})=I(\phi)=\frac{1}{4 L} \int_{Q_{p}}|\nabla \phi|^{2} d \mathbf{x} .
$$

Indeed, for the minimizer $\phi$ of (2.3) the Euler-Lagrange equations are (2.5). Multiply (2.5 a) by $\phi$ and integrate by parts.

$$
0=-\int_{Q_{p}}|\nabla \phi|^{2} \delta \mathbf{x}+\int_{y= \pm 1} \partial \phi / \partial \mathbf{n} \phi \delta \mathbf{x}+\sum_{i=1}^{N} t_{i} \int_{\partial D_{i}} \partial \phi / \partial \mathbf{n} \delta \mathbf{x} .
$$

By $(2.5$ c) we have

$$
\int_{Q_{p}}|\nabla \phi|^{2} \delta \mathbf{x}=\int_{y=1} \partial \phi / \partial \mathbf{n} \delta \mathbf{x}-\int_{y=-1} \partial \phi / \partial \mathbf{n} \delta \mathbf{x} .
$$

By (2.6) we have

$$
\frac{1}{2} \int_{Q_{p}}|\nabla \phi|^{2} d \mathbf{x}=\int_{y=1} \partial \phi / \partial \mathbf{n} d \mathbf{x}=2 L \widehat{a} .
$$

Using the Legendre transform (see [12]) the dual variational formulation is obtained as follows. Using ( $2.5 \mathrm{a}$-c) let us define the dual space $W_{p}$.

$$
W_{p}=\left\{\mathbf{v} \in L_{2}\left(Q_{p}\right): \mathbf{v}( \pm L, y) \cdot \mathbf{n}=0, \int_{\partial D_{i}} \mathbf{v} \cdot \mathbf{n} d \mathbf{x}=0, \nabla \cdot \mathbf{v}=0\right\}
$$


For any fixed $\phi \in V_{p} \mathbf{v}$ is defined via the Legendre transform

$$
\mathbf{v}=\arg \max _{\tilde{\mathbf{v}} \in W_{p}} \int_{Q_{p}}\left(\tilde{\mathbf{v}} \cdot \nabla \phi-\frac{1}{2}|\tilde{\mathbf{v}}|^{2}\right) d \mathbf{x}
$$

The dual variational principle for the effective conductivity (see [12]) is

$$
\widehat{a}=\frac{1}{2 L} \max _{\tilde{\mathbf{v}} \in W_{p}}\left\{\int_{y=1} \tilde{\mathbf{v}} \cdot \mathbf{n} d \mathbf{x}-\int_{y=-1} \tilde{\mathbf{v}} \cdot \mathbf{n} d \mathbf{x}-\frac{1}{2} \int_{Q_{p}} \tilde{\mathbf{v}}^{2} d \mathbf{x}\right\} .
$$

Hence for any $\phi \in V_{p}$ and $\mathbf{v} \in W_{p}$ we have bounds

$$
\frac{1}{2 L}\left[\int_{y=1} \mathbf{v} \cdot \mathbf{n} d \mathbf{x}-\int_{y=-1} \mathbf{v} \cdot \mathbf{n} d \mathbf{x}-\frac{1}{2} \int_{Q_{p}} \mathbf{v}^{2} d \mathbf{x}\right] \leq \widehat{a} \leq \frac{1}{4 L} \int_{Q_{p}}|\nabla \phi|^{2} d \mathbf{x} .
$$

Moreover, the upper bound equals the lower bound in (2.16) if $\mathbf{v}=\nabla \phi$.

\section{Construction of the discrete network}

\subsection{Effective conductivity for periodic square and hexagonal lat- tices}

We start the section on the discrete network model with the comparison of effective properties of the composites with two different periodic structures of inclusions - hexagonal and square, because it motivates the need for the discrete network approach. A composite with the periodic square lattice of disks was analyzed by J.B. Keller. In [14] an asymptotic formula for the effective conductivity was derived when the disks are closely spaced. Here we give the main idea of the heuristic argument and the final result from [14]. On the other hand, a composite with the periodic hexagonal lattice plays an important role for applications, because the densest packing in two dimensions is achieved for hexagonal packings. For the case of the periodic hexagonal lattice we apply the idea from [14] and discuss it in more detail. Then we compare our value of the effective conductivity for the hexagonal lattice with the value of the effective conductivity for the square lattice from [14]. The error analysis that we give later in this paper rigorously justifies these computations.

The main idea of the computation of the effective conductivity for the square lattice is based on the observation that the fluxes $\nabla \phi$ are significant only in the areas (necks) between closely spaced disks, and in these necks the fluxes can be easily computed. For two disks $D_{i}$ and $D_{j}$ the potential $\phi$ in the neck $\Pi_{i j}$ between them is approximately a linear interpolation between the potentials $t_{i}$ and $t_{j}$ on disks $D_{i}$ and $D_{j}$ respectively. More specifically, consider just two disks $D_{i}$ and $D_{j}$ in the whole space $\mathbb{R}^{2}$ (figure 3.1). Let $\delta_{i j}$ be the distance between the disks. There is an exact solution $\phi$ to the Laplace equation (see [22]) in $\mathbb{R}^{2}$. The local flux $\nabla \phi$ of this solution in the neck $\Pi_{i j}$ between $D_{i}$ and $D_{j}$ is singular asymptotically as $\delta_{i j} \rightarrow 0$. The main asymptotic term is proportional to the potential drop $t_{i}-t_{j}$ and inversely proportional to the distance between the disks. More specifically, if we align the neck $\Pi_{i j}$ between two neighbors 
$D_{i}$ and $D_{j}$ with the vertical direction as indicated on figure 3.1, then by the linear interpolation the local flux in $\Pi_{i j}$ satisfies

$$
\begin{aligned}
& \nabla \phi=\left(0, \frac{t_{i}-t_{j}}{H(x)}\right)+\text { Const }, \\
& H(x)=\delta_{i j}+R_{i}+R_{j}-\sqrt{R_{i}^{2}-x^{2}}-\sqrt{R_{j}^{2}-x^{2}},
\end{aligned}
$$

where $R_{i}$ is the radius of $D_{i}$. We assume that $R_{i}=\infty$ for any boundary segment. If the distance between the disks $\delta_{i j} \rightarrow 0$, then $H(x)=O\left(\delta_{i j}\right)$ and therefore the local flux between the disks is singular.

$$
\nabla \phi=\left\{\begin{array}{l}
\left(0, \frac{t_{i}-t_{j}}{H(x)}\right)+O(1) \text { in } \Pi_{i j} \text { as } \delta_{i j} \rightarrow 0, \\
O(1), \text { if } \delta_{i j} \geq \text { Const. }
\end{array}\right.
$$

Hence if the neck $\Pi_{i j}$ is wide enough and the disks are close then the total flux through this neck is also singular. If we align the neck $\Pi_{i j}$ with the vertical direction as indicated on figure 3.1, then asymptotically

$$
\left(\int_{\Pi_{i j}}\left|\frac{\partial \phi}{\partial x}\right| d x d y, \int_{\Pi_{i j}}\left|\frac{\partial \phi}{\partial y}\right| d x d y\right)=\left(0, g_{i j}^{0}\left(t_{i}-t_{j}\right)\right)+O(1) \text {, as } \delta_{i j} \rightarrow 0,
$$

where $g_{i j}^{0}$ is the specific flux.

Definition 2 For any two disks centered at $x_{i}$ and $x_{j}$ with radii $R_{i}$ and $R_{j}$ respectively the specific flux

$$
g_{i j}^{0}=\int_{S_{1}}^{S_{2}} \frac{d x}{H(x)}
$$

where the neck widths $S_{1}$ and $S_{2}$ are as defined on figure 3.1.

For any fixed neck width as $\delta_{i j} \rightarrow 0$ the specific flux can be approximated by a nondimensional parameter - the relative interparticle flux.

Definition 3 For any two disks centered at $x_{i}$ and $x_{j}$ with radii $R_{i}$ and $R_{j}$ respectively the relative interparticle flux

$$
g_{i j}=\pi \frac{\sqrt{\frac{2 R_{i} R_{j}}{R_{i}+R_{j}}}}{\sqrt{\delta_{i j}}}, \text { where } \delta_{i j}=\left|x_{i} x_{j}\right|-R_{j}-R_{i}
$$

is the (Euclidean) distance between the disks. In particular, the relative flux between a disk with radius $R_{i}=R$ and a boundary line (with radius $R_{j}=\infty$ ) is

$$
g_{i j}=\pi \sqrt{\frac{2 R}{\delta_{i j}}} .
$$

The relative flux between two disks with equal radii $R_{i}=R_{j}=R$ is

$$
g_{i j}=\pi \sqrt{\frac{R}{\delta_{i j}}} .
$$


The asymptotic equivalence

$$
g_{i j}^{0}=g_{i j}+O(1) \text { as } \delta_{i j} \rightarrow 0
$$

for the case of equal disks and for the case of a disk and the boundary is shown in Appendix A.3. For the case of two disks with arbitrary radii see [4].

Assuming that the behavior of the effective conductivity of this medium mainly depends upon the fluxes between "neighbors" (closely spaced disks) the asymptotic formula of effective conductivity of a periodic square lattice of two-dimensional disks is (see [14])

$$
\widehat{a}_{\text {square }}=\frac{\pi}{2} \sqrt{\frac{R}{\delta}}+O(1), \text { as } \delta \rightarrow 0 .
$$

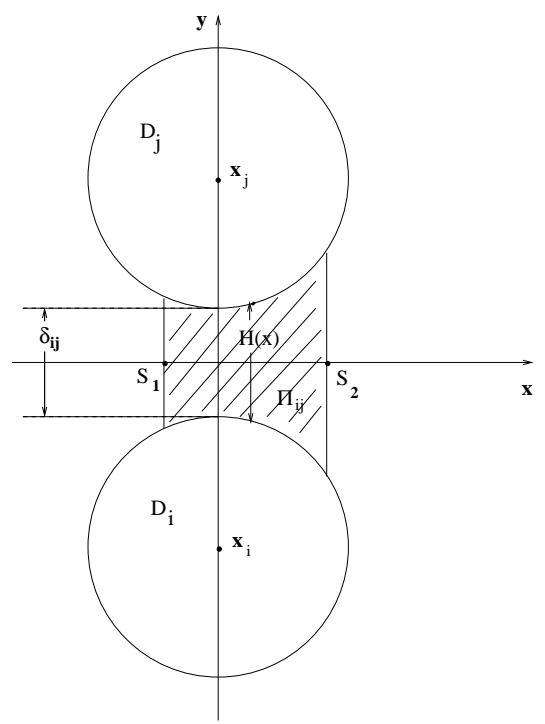

Figure 3.1: The hatched region is the neck between two neighbors

The asymptotic formula for the periodic hexagonal lattice can be computed using the same idea of [14]. More specifically, in order to compute the effective conductivity of a medium with closely spaced disks, one needs to know the values of the potential $\phi$ on the disks and to determine which disks are closely spaced. Let us implement this idea. Consider the situation when the disks are perfectly aligned in layers in the horizontal direction. There are two possible cases of such an alignment: the first alignment is shown on figure 3.2, the second one can be obtained by the rotation of figure 3.2 by $\pi / 2$. However, due to the hexagonal symmetry of the problem the tensor of effective conductivity is the same in both cases. Hence we consider only the case shown on figure 3.2.

Suppose there are $N$ disks in a horizontal layer, $N \gg 1$. Suppose that there are $M+1$ layers, $M \gg 1$. For ease of presentation assume that at the vertical boundary we have periodic boundary conditions and assume that the horizontal boundaries 


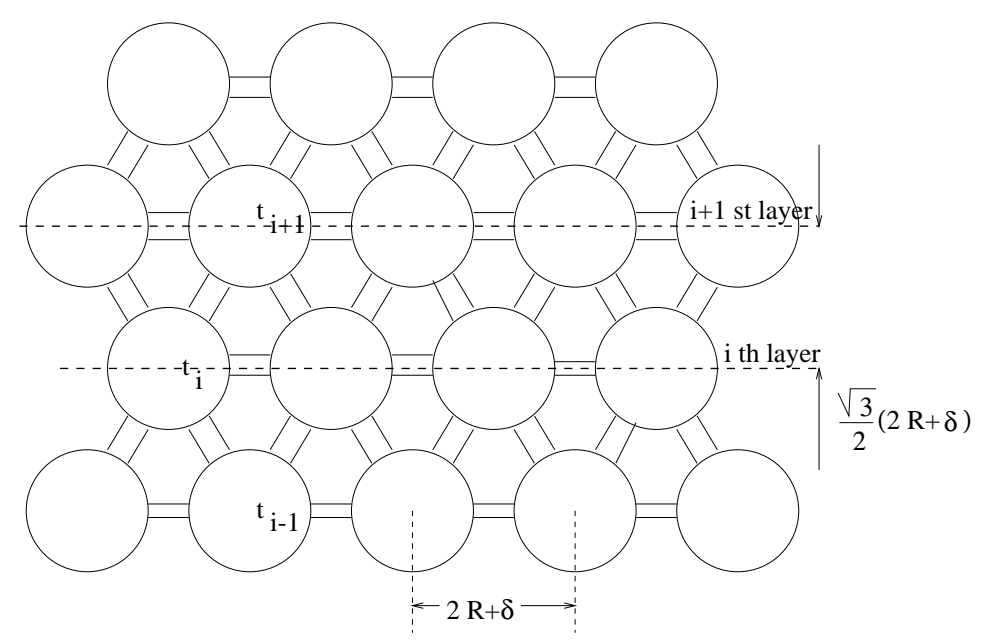

Figure 3.2: Hexagonal lattice of inclusions.

bisect through the top and the bottom layers of the disks respectively. The boundary conditions on horizontal boundaries are determined by $(2.5(\mathrm{e}))$.

The height and the width of the composite are respectively

$$
2=\sqrt{3} / 2(2 R+\delta) M, 2 L=(2 R+\delta) N .
$$

Let us find the values $t_{i}$ on disks $D_{i}$ of the solution $\phi$ explicitly. Using symmetry of the distribution of the disks we conclude that if $\phi$ is the solution of (2.5) then all $t_{i}$ should be the same in every horizontal layer, and the potential drop between any two consecutive layers is the same along the composite, and since the potential at the bottom boundary is -1 and it is 1 at the top boundary we have

$$
t_{i+1}-t_{i}=\frac{2}{M} .
$$

Since there are no fluxes in the horizontal necks, we need to keep track of non-horizontal necks only. There are exactly two necks going up from each disk. Summing up over all these non-horizontal necks and using (3.7) we have

$$
\widehat{a}=\frac{1}{4 L} \sum_{i=1}^{M} 2 N \pi \sqrt{\frac{R}{\delta}}\left(\frac{2}{M}\right)^{2}+O(1)=\frac{1}{L} 2 \pi \sqrt{\frac{R}{\delta}} \frac{N}{M}+O(1) \text { as } \delta \rightarrow 0 .
$$

or simply

$$
\widehat{a}=\sqrt{3} \pi \sqrt{\frac{R}{\delta}}+O(1) \text { as } \delta \rightarrow 0 .
$$

The error estimates we prove in section 4.3 imply that

- our choice of boundary conditions is not restrictive, general boundary conditions $(2.5, \mathrm{~b}, \mathrm{e})$ give $O(1)$ corrections to the effective conductivity as $\delta \rightarrow 0$; 
- our asymptotic computations are rigorous.

Therefore we have proved

Theorem 3.1 For the two-dimensional hexagonal periodic lattice of disks with radius $R$ and the distance between neighboring disks $\delta$ the effective conductivity as $\delta \rightarrow 0$

$$
\widehat{a}_{h e x}=\sqrt{3} \pi \sqrt{\frac{R}{\delta}}+O(1) .
$$

Several fundamental observations can be made based on the comparison of the results of computations of the effective conductivity of square and hexagonal lattices. First, in contrast to the case of not highly packed particular composites, here the volume fraction of the inclusions is not a characteristic parameter. Indeed, suppose the volume fraction of disks equals to $\pi / 4$. By (3.9) and (3.11) for the same volume fraction of disks

$$
\widehat{a}_{\text {hex }}=\text { Const }<\infty \text {, but } \widehat{a}_{\text {square }}=\infty .
$$

Such qualitative difference comes from the fact that in two dimensions the most efficient packing of disks is hexagonal. Suppose $f$ is the volume fraction of the disks. Then for square packing

$$
f_{\text {square }}=\frac{\pi}{4} /\left(1+\frac{\delta}{2 R}\right)^{2} \approx \frac{\pi}{4}\left(1-\frac{\delta}{R}\right),
$$

and for hexagonal packing

$$
f_{\mathrm{hex}}=\frac{\pi \sqrt{3}}{6} /\left(1+\frac{\delta}{2 R}\right)^{2} \approx \frac{\pi \sqrt{3}}{6}\left(1-\frac{\delta}{R}\right) .
$$

The maximal value of $f$ is $\pi / 4$ for square packing, and maximal value of $f$ is $\pi \sqrt{3} / 6$ ( $\pi \sqrt{3} / 6>\pi / 4$ ) for hexagonal packing. Second, for high contrast, highly packed particular composites the effective conductivity is determined by the the geometry of the disks' array. This indicates that the relative interparticle distance $\delta / R$ may be the appropriate characteristic parameter. Indeed, both formulas (3.9) and (3.11) depend on the relative interparticle distance:

$$
\widehat{a}=\mathbf{C}_{\mathbf{0}} \sqrt{\frac{R}{\delta}}+O(1)
$$

but the constant $\mathbf{C}_{\mathbf{0}}$ depends on the number of necks connecting closely spaced disks. Third, in the case of square and hexagonal packings there is asymptotic equivalence between the continuum minimization problem (2.4) and its discrete analog

$$
\frac{1}{4 L} \min _{\phi} \int_{Q_{p}}|\nabla \phi|^{2} d \mathbf{x}=\frac{1}{4 L} \min _{t_{i}} \sum_{\Pi_{i j}} g_{i j}\left(t_{i}-t_{j}\right)^{2}+O(1), \text { as } \delta \rightarrow 0 .
$$

Here $g_{i j}$ are all the same and they are determined by (3.7). Moreover, the solutions $\mathbf{t}=\left(t_{1}, t_{2} \ldots, t_{N}\right)$ of this discrete minimization problem in the case of square and 
hexagonal packings are identical to the corresponding values $t_{i}$ on the disks $D_{i}$ determined by the original continuum problem (2.4). Therefore, in the case of square and hexagonal periodic distributions of disks in order to capture the main asymptotic term of the effective conductivity it is sufficient to study the discrete minimization problem for the associated discrete network:

$$
\frac{1}{4 L} \min _{t_{i}} \sum_{\Pi_{i j}} g_{i j}\left(t_{i}-t_{j}\right)^{2} .
$$

The discrete network has very few parameters, hence it is attractive for numerical simulations. Indeed, in both cases this discrete network consists of

vertices $x_{i}$, these are centers of disks $D_{i}$;

edges $e_{i j}, \quad$ these are necks between closely spaced disks $D_{i}$ and $D_{j}$;

lengths $\delta_{i j}$, these are lengths of necks $e_{i j}$.

In terms of these three parameters the solution to the discrete minimization problem (hence the leading term in asymptotics of the effective conductivity) can be computed.

\subsection{The discrete network for densely packed high contrast com- posites}

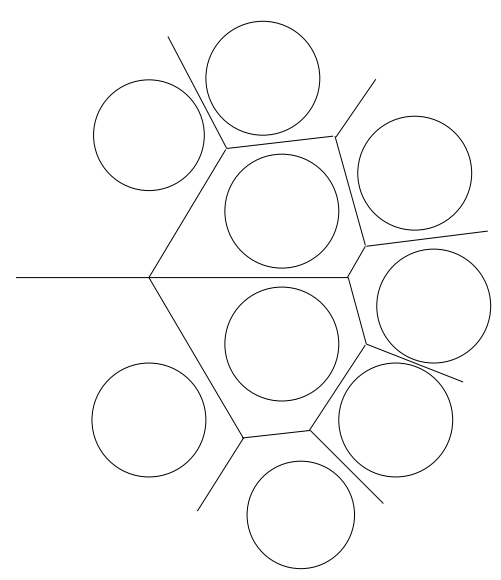

(a) Voronoi tessellation

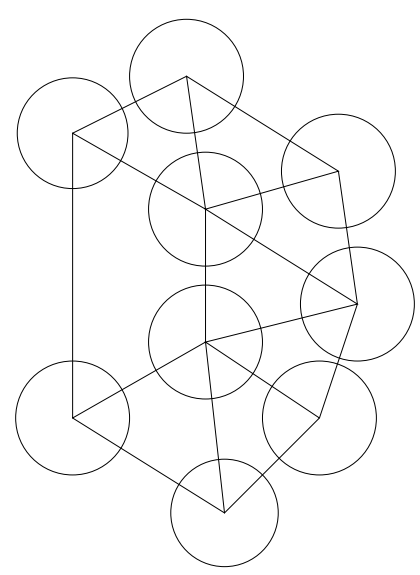

(b) Delaunay graph

Figure 3.3: Voronoi tessellation and Delaunay graph

The discrete network is basically the Delaunay graph (triangulation) of the domain $\Pi$ with respect to the centers of the disks. Here we describe the construction of the discrete network and give some definitions, that we use in the rest of the paper.

Intuitively, we mimic here the idea of the discrete network (3.13) for the case of irregularly distributed disks. The main advantage of the construction is that by purely 

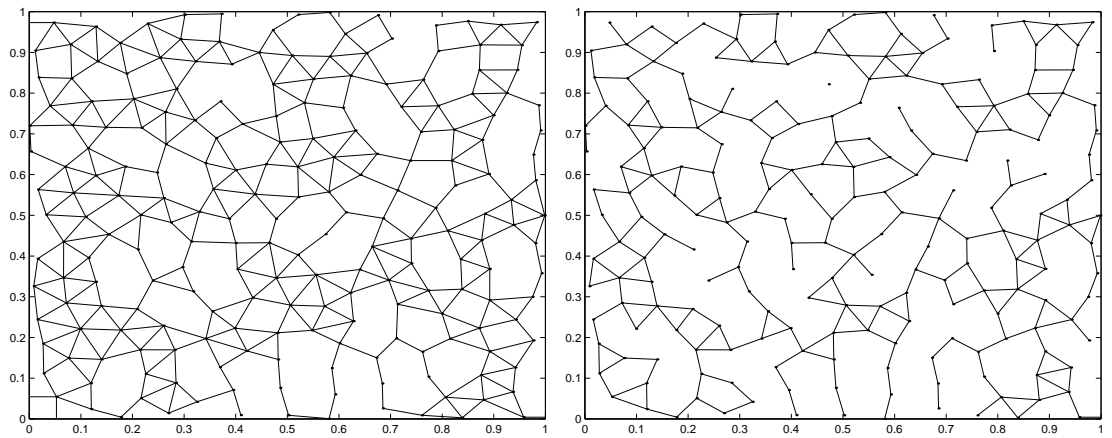

.03-subgraph

.015 -subgraph
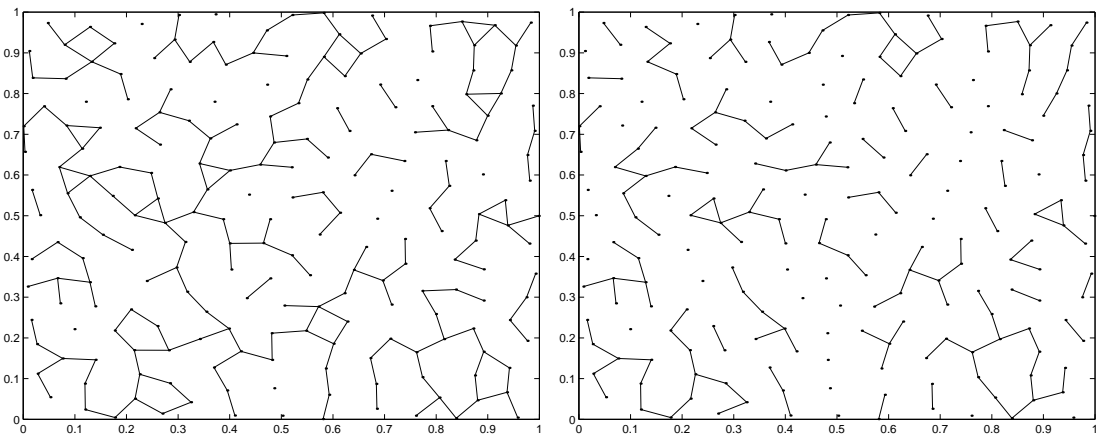

.007 -subgraph

.003 -subgraph

Figure 3.4: Connectivity patterns of $\delta$-subgraphs

geometrical means we determine which disks should be connected by a neck. The construction relies on the notion of neighbors. In order to give the precise meaning to the notion of neighbors for a random distribution of disks we introduce here the Voronoi tessellation and the dual Delaunay triangulation. By means of the Voronoi tessellation for each disk $D_{i}$ we can uniquely define its neighbors.

Recall the definition of the Voronoi tessellation.

Definition 4 For in a given distribution of vertices $x_{i}, i=1, \ldots, N$ in the twodimensional rectangular domain $\Pi=[-L, L] \times[-1,1]$ the Voronoi cell $V_{i}$ associated with $x_{i}$ is a polygon, that consists of all the points in $\Pi$ at least as close to $x_{i}$ as to any other vertex. The set of all such Voronoi cells $V_{i}$ is the Voronoi tessellation of $\Pi$.

Consider the set of the centers $x_{i}, i=1,2, \ldots, N$ of all the disks $D_{i}$. Construct the Voronoi tessellation for the vertices $x_{i}, i=1,2, \ldots, N$. Construct the dual Delaunay graph (triangulation), that is connect every pair of vertices $x_{i}$ and $x_{j}$ by the edge $e_{i j}$ if they share a common edge in the Voronoi tessellation (see figure 3.3). Typically if any two Voronoi cells share a common vertex then they share a common edge as well. The case when more than three Voronoi cells share a common vertex is exceptional for randomly distributed disks, therefore the Delaunay graph is usually a triangulation. 
However, we include in our analysis the case when more than three cells share a common vertex (see e.g. the leftmost vertex of the Voronoi tessellation on figure 3.3). The boundary of $\Pi$ needs an additional construction. If for a vertex $x_{i}$ its Voronoi cell $V_{i}$ is adjacent to the boundary (that is one of the sides of $V_{i}$ lies on the boundary of $\Pi$ ) and the radius of the disk $R_{i}=R$ is smaller than the distance from $x_{i}$ to this boundary, then connect $x_{i}$ with this boundary by the perpendicular line. Denote the intersection of this perpendicular and the boundary by $x_{i^{\prime \prime}}, i^{\prime \prime}>N$ and the line segment between $x_{i}$ and $x_{i^{\prime \prime}}$ by the edge $e_{i i^{\prime \prime}}$.

Remark 1 Note, that while constructing this (modified) Delaunay graph we have added additional vertices $x_{i^{\prime \prime}}, i^{\prime \prime}>N$ that lie on the boundary of $\Pi$. For the uniformity of the presentation we will define such vertices as centers of quasidisks with radius $R=\infty$ in section 3.3 .

Definition 5 For a given distribution of disks $D_{i}$ with centers $x_{i} i=1, \ldots, N$ the discrete network $\mathbb{D}$ is set of vertices $x_{i} i=1, \ldots, M, M \geq N$ and edges $e_{i j}$ of the (modified) Delaunay graph.

Definition 6 A vertex $x_{i}$ is an upper (lower/left/right) boundary vertex, if $x_{i}$ lies on the upper (lower/left/right) boundary or $x_{i}$ is the center of the disk $D_{i}$, that intersects the upper (lower/left/right) boundary. The set $S^{+}\left(S^{-} / S^{l} / S^{r}\right)$ is the set of upper (lower/left/right) boundary vertices.

Definition 7 Any two disks $D_{i}$ and $D_{j}$ are said to be neighbors, if their centers $x_{i}$ and $x_{j}$ are connected by the edge $e_{i j}$.

Definition 8 The length $\delta_{i j}$ of an edge $e_{i j}$ is the (Eulidean) distance between the disks $D_{i}$ and $D_{j}$.

$$
\delta_{i j}=\left\{\begin{array}{cc}
d_{i j}-2 R, & \text { if } x_{i} \text { and } x_{j} \text { are centers of disks } D_{i} \text { and } D_{j} \\
d_{i j}-R, & \text { otherwise, that is if one of the disks }
\end{array}\right.
$$

here $d_{i j}=\left|x_{i}-x_{j}\right|$ is the (Euclidean) distance between $x_{i}$ and $x_{j}$.

Note, that if two disks $D_{i}$ and $D_{j}$ are closely spaced $\left(\delta_{i j}<<R\right)$, then they are neighbors. The converse statement is not true - two disks $D_{i}$ and $D_{j}$ can be neighbors, but $\delta_{i j} \sim R$. Therefore from the physical point of view, if there is a conducting spanning cluster of inclusions in the composite then the effective conductivity of the composite should depend on essential edges, which are sufficiently small in length. The physical notion of a conducting spanning cluster can be defined as a path on the graph $\mathbb{D}$ such that this path connects the top and the bottom boundaries of the domain $\Pi$ and the distance $\delta_{i j}$ between every two consecutive vertices of this path $\delta_{i j} \leq \delta$ where $\delta$ is sufficiently small. The physical notion of the essential edges can be defined by just by picking a small $\delta$ and saying that an edge $e_{i j}$ is essential, if its length $\delta_{i j} \leq \delta$. Then these essential edges form a subgraph of our original discrete network graph. Depending on how small the $\delta$ is, this $\delta$-subgraph may have only few edges for very small $\delta$ or 
a lot of edges if $\delta$ is sufficiently large (see figure (3.4)). In particular, the 2-subgraph is the whole discrete network, because the distance to the boundary for any interior disk is always $<2$. On the other hand there is a small $\delta_{0}$, so that the $\delta_{0}$-subgraph does not have any edges at all. If $\delta_{1}<\delta_{2}$, then the $\delta_{1}$-subgraph is nested in the $\delta_{2}$ subgraph, that is every edge of the $\delta_{1}$-subgraph is also an edge in the $\delta_{2}$-subgraph, but not vice-versa. The 2-subgraph has the largest number of edges among all the $\delta$ subgraphs. For the 2-subgraph vertices of every pair of neighbors are connected, and the 2-subgraph typically triangulates the domain $\Pi$. When $\delta<2$, this is not necessarily true any more; however, when $\delta$ is not very small the $\delta$-subgraph still partitions the domain into polygons. The size of these polygons measures the connectivity properties of in the composite: if these polygons have a lot of edges, then the connectivity properties of the composite are poor; if, on the other hand, these polygons have few edges, then the connectivity properties of the composite are good. In the latter case using the intuition from the case of periodic square and hexagonal lattices we expect, that the fluxes between the closely spaced disks will play the dominant role in the computation of the effective conductivity. Hence we wish to study these connectivity properties of $\delta$-subgraphs. The graph-theoretical concept of the minimal cycles accurately captures the idea of the size of the polygons in the partition of $\Pi$ by the $\delta$-subgraph. A minimal cycle on a (sub)graph is a closed path, that cannot be shortened by replacing some edges of this path by other edges of the (sub)graph. As usual, the situation at the boundary needs a special treatment. At the boundary we need to consider "boundary" paths. These are the paths, that start on the boundary and end on the boundary. For a boundary path a minimal cycle is again the one that cannot be shortened. These connectedness properties of $\delta$-subgraphs are used in the in section 4 for error estimates of the discrete network approximation. Therefore, for completeness, in the rest of this section we give the precise graph-theoretical definitions related to this notion. Most of them are taken from [5].

Definition 9 For any $\delta>0$ the $\delta$-subgraph $\mathbb{D}_{\delta}$ of the discrete network (graph) $\mathbb{D}$ is the subset of edges $e_{i j}$ and their incident vertices $x_{i}$ and $x_{j}$ of $\mathbb{D}$, such that their length $\delta_{i j} \leq \delta$. For any subgraph a vertex is incident if it is an endvertex of one of its edges.

Definition 10 A path of a $($ sub $)$ graph $\mathbb{D}_{\delta}$ from $x_{0}$ to $x_{n}$ is an alternating sequence of

$$
x_{0}, e_{01}, x_{1}, e_{12}, x_{2}, e_{23}, \ldots, x_{n-1}, e_{n-1 n}, x_{n},
$$

of distinct vertices $x_{i}$ and edges $e_{i j}$. Such a path has size $\mathbf{n}$, the vertices $x_{0}$ and $x_{n}$ are said to be the endvertices. The vertices $x_{1}, \ldots, x_{n-1}$ are said to be the interior vertices.

Conventionally (see [5]), the size of a path is called its length. We do not use here this standard notation, because the following definition of the length of a path is more natural in our setting (due to definition 8).

Definition 11 The length of a path is the sum of lengths of its edges as defined in definition 8. 
Definition 12 An internal cycle $C$ of $\mathbb{D}_{\delta}$ is an alternating sequence of

$$
x_{0}, e_{01}, x_{1}, e_{12}, x_{2}, e_{23}, \ldots x_{n}, e_{n 0}, x_{0},
$$

of vertices and edges, such that

$$
x_{0}, e_{01}, x_{1}, e_{12}, x_{2}, e_{23}, \ldots x_{n}
$$

is a path, and $e_{n 0}$ connects the vertices $x_{0}$ and $x_{n}$. Such a cycle has size $\mathbf{n}+\mathbf{1}$.

Definition 13 A boundary cycle $C$ of $\mathbb{D}_{\delta}$ is a path

$$
x_{0}, e_{01}, x_{1}, e_{12}, x_{2}, e_{23}, \ldots x_{n}
$$

such that the endvertices $x_{0}$ and $x_{n}$ lie on the boundary of the domain $\Pi$. Such a boundary cycle has size $\mathbf{n}$.

Note that the endvertices $x_{0}$ and $x_{n}$ of a boundary cycle may lie on different parts of the boundary of the domain $\Pi$, for example left and upper.

Definition 14 A minimal cycle $C_{m}$ is a (internal or boundary) cycle, such that for any two vertices $x_{i}$ and $x_{j}$ of this cycle the shortest path from $x_{i}$ to $x_{j}$ is a subset of the cycle $C_{m}$. If the cycle $C_{m}$ is a boundary cycle, we also require that for any interior point $x_{i}$ of this cycle the shortest path from to $x_{i}$ to any point $x_{k}$ on the boundary is a subset of the cycle $C_{m}$.

Note that we require for a minimal boundary cycle an additional condition. This condition guarantees that a boundary cycle cannot be shortened by connecting an interior point of this cycle with the boundary.

Definition 14 is a formalization of an intuitive notion of a hole in a composite. Each hole in a composite is surrounded by a loop of conducting disks (see figure 1.1 (a)). On the modified Delaunay graph this loop corresponds to an $\mathcal{N}$-gon, which is a minimal cycle of this graph.

Definition 15 The size of the largest minimal cycle $\mathcal{N}$ of a (sub)graph $\mathbb{D}_{\delta}$ is the upper bound on the size of all its minimal cycles, that is

$$
\mathcal{N}=\max _{C_{m} \subset \mathbb{D}_{\delta}} \operatorname{size}\left(C_{m}\right)
$$

where $C_{m}$ is a minimal cycle.

Definition 16 The interior of a cycle $C$ is a polygon having the cycle $C$ as its boundary, that is $\partial \operatorname{Int}_{C}=C$.

The degree of the connectedness of the whole graph $\mathbb{D}$ can now be quantified in terms of the two parameters: $\delta$ and $\mathcal{N}$, and an a priori relative error estimate for the discrete network approximation $\mathbb{D}$ will be determined in terms of these parameters only.

Definition 17 A discrete network (graph) $\mathbb{D}$ is $\delta$ - $\mathcal{N}$ connected if 
(i) for every vertex $x_{i}$ of the graph $\mathbb{D}$ there exists a minimal cycle $C$ of its $\delta$-subgraph $\mathbb{D}_{\delta}$ such that $x_{i} \in$ Int $_{C}$, where Int $C$, the interior of a cycle $C$.

(ii) the size of the largest minimal cycle of $\mathbb{D}_{\delta}$ is $\mathcal{N}$.

Condition (i) in definition (17) implies that if $R, \mathcal{N}$ and $\delta$ are not too large, then there exists at least one path on the graph $\mathbb{D}_{\delta}$ such that it connects the top and the bottom boundaries of the domain $\Pi$. This can be shown by contradiction using the duality argument. Suppose there are no paths on the graph $\mathbb{D}_{\delta}$ that connect the top and the bottom boundaries. Then there exists a path in the whole domain $\Pi$ such that it connects the left and the right boundaries of $\Pi$ and does not intersect $\mathbb{D}_{\delta}$. The length of this path on the one hand must be larger than the distance between the left and the right boundaries, on the other hand it cannot be larger than the diameter of the largest minimal cycle. Recall that the distance between any two vertices in $\mathbb{D}$ does not exceed $2 R+\delta$, hence the inequality the inequality

$$
2 L \leq(2 R+\delta) \mathcal{N} / 2
$$

must hold. But inequality (3.16) cannot hold if $R, \mathbb{N}$ and $\delta$ are not too large, therefore we have a contradiction. The existence of a path on $\mathbb{D}_{\delta}$ which connects the top and the bottom boundaries means the existence of the conducting spanning cluster, since the distance $\delta_{i j}$ between every two consecutive vertices of this path satisfies $\delta_{i j} \leq \delta$ by definition of $\mathbb{D}_{\delta}$.

\subsection{The triangle-neck partition}

Here we describe an algorithm that leads to a construction of the partition of the domain $Q_{p}$ (defined by (2.1)) into non-overlapping subdomains. This algorithm is the triangle-neck partition of the domain $Q_{p}$ induced by the Voronoi tessellation. It is an auxiliary construction, which is used in section 4 as a convenient and efficient tool for the construction of the trial functions for the error estimates. The main goal is to have a decomposition of $Q_{p}$ into simple geometric figures - necks and triangles only, such that necks between neighbors must be included into the decomposition. By means of this decomposition we will able to account for the idea of Keller that the flux is important only in necks between almost touching disks. This decomposition also makes the derivation of variational error estimates transparent.

The main idea of the triangle-neck partition is to connect two neighbors $D_{i}$ and $D_{j}$ by a neck $\Pi_{i j}$. The widths of these necks are chosen so that after we connect all the neighbors, the domain $\Pi$ is partitioned into necks $\Pi_{i j}$ and polygons. These polygons are typically triangles. If they are not triangles, we partition them further by essentially drawing diagonal lines.

Here is the precise algorithm. For a given distribution of disks $D_{i}, i=1, \ldots, N$ construct the Voronoi tessellation and the discrete network $\mathbb{D}$ as in section 3.2. Consider any two neighbors $D_{i}$ and $D_{j}$ with centers $x_{i}$ and $x_{j}$ respectively (see figure 3.5(a)). Denote by $O_{n}$ and $O_{p}$ the endpoints of the common edge of their Voronoi cells $V_{i}$ and $V_{j}$. For each $j$ connect the center $x_{j}$ with all the vertices of its Voronoi cell $V_{j}$ by auxiliary line segments (see dotted lines on figure 3.5(a)). Denote by $A_{j n}$ the 


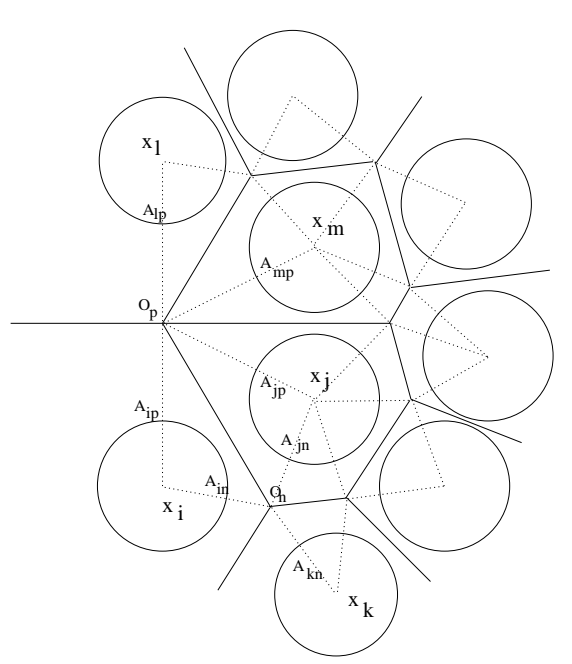

(a) Voronoi tessellation

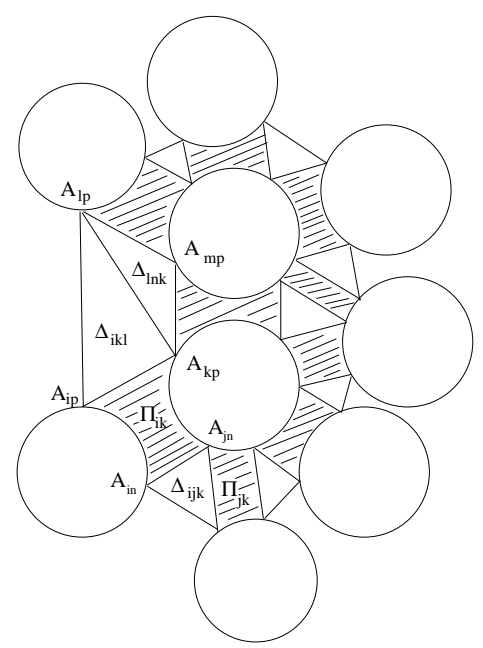

(b) Triangle-neck partition

Figure 3.5: Decomposition of Voronoi cell

intersection of the line segment $x_{j} O_{n}$ with the circumference of the disk $D_{j}$. Define similarly points $A_{i n}$ and $A_{k n}$. Connect the points $A_{i n}, A_{j n}$ and $A_{k n}$.

Definition 18 The neck $\Pi_{i j}$ between the neighbors $D_{i}$ and $D_{j}$ is the curvilinear quadrangle $A_{i n} A_{i p} A_{j p} A_{j n}$, bounded by the two line segments $A_{i n} A_{j n}$ and $A_{i p} A_{j p}$ and the two $\operatorname{arcs} A_{i n} A_{i p}$ and $A_{j n} A_{j p}$.

Typically a neck $\Pi_{i j}$ is not symmetric with respect to the line connecting the centers of the disks $D_{i}$ and $D_{j}$. An example of a neck is given on figure 3.1 where we used the local coordinate system where the centers of the both disks lie on the $y$-axis. In this coordinate system the width of the left half-neck is $\left|S_{1}\right|, S_{1}<0$, and the width of the right half-neck is $\left|S_{2}\right|, S_{2}>0$. Note that inequalities $S_{1}<0 S_{2}>0$ are not true in general, but $S_{1} \leq S_{2}$ always by our construction.

Definition 19 The maximal and the minimal relative half-neck widths are defined by

$$
\beta_{i j}^{\max }=\max \left(\frac{\left|S_{1}\right|}{R}, \frac{\left|S_{2}\right|}{R}\right), \beta_{i j}^{\min }=\min \left(\frac{\left|S_{1}\right|}{R}, \frac{\left|S_{2}\right|}{R}\right), 0 \leq \beta_{i j}^{\min } \leq \beta_{i j}^{\max }<1 .
$$
4 .

We use the relative half-neck widths $\beta_{i j}^{\max }$ and $\beta_{i j}^{\min }$ in the error estimates in section

When we apply this algorithm to all neighbors, in general, all these line segments $A_{i n} A_{j n}$ partition the domain $Q_{p}$ into necks $\Pi_{i j}$ between neighboring disks and triangles $\Delta_{i j k}$ (see figure 3.5(b)). In degenerate (exceptional) cases instead of triangles we obtain polygons (for example quadrangle $A_{i p} A_{l p} A_{m p} A_{k p}$ on figure 3.5(b)) which can 
be further partitioned into triangles. We illustrate the degenerate case by an example of a periodic square lattice. In this case our algorithm leads to the partition into necks and squares (see figure 3.6). We draw a diagonal in each square to partition these squares into triangles (the dashed lines on figure 3.6). Hence our algorithm in all the cases provides with the partition of $Q_{p}$ into necks and triangles only.

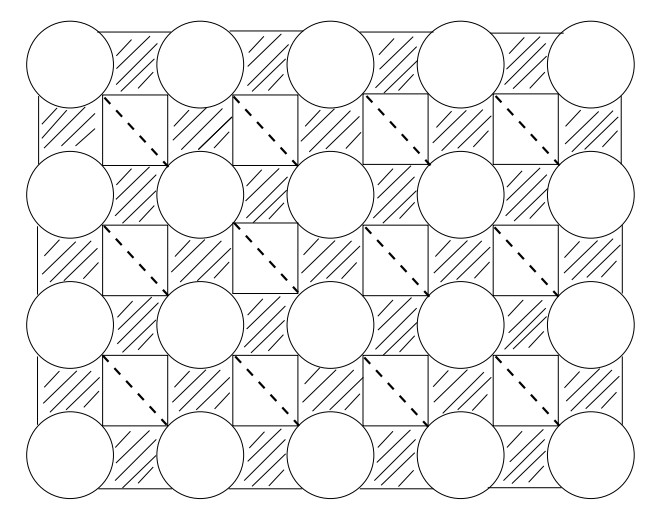

Figure 3.6: Necks are hatched. Dashed lines partition squares into triangles.

The situation at the boundary $y= \pm 1$ and $x= \pm L$ again needs a special treatment. For the construction of the partition of $Q_{p}$ near the boundary we use reflections about all four parts of it (upper, lower, right and left boundary). Recall that all the centers $x_{i}$ of the disks lie inside the domain $\Pi$, hence the centers of the reflected disks will always lie outside the domain $\Pi$. The case when the disks lie outside the boundary can also be treated by the model; however, it requires unnecessary technical complications.

Consider, for example, the left boundary $x=-L$. The algorithm is shown on figure 3.7. Reflect symmetrically along the line $x=-L$ all the disks including the disks that lie on the boundary. Disks that lie on the boundary partially overlap with the "ghost" disks (dotted disks on figure 3.7) which are their mirror images; however, for the distribution of original disks and the ghost disks we can still apply the Voronoi tessellation and the algorithm proposed for the interior disks.

Introduce a notion of a quasidisk.

Definition 20 A quasidisk $D_{i^{\prime \prime}}$, is the part of a neck $\Pi_{i i^{\prime}}$ that lies on the boundary of П. The radius of a quasidisk is $\infty$.

An example is the line segment $C D \equiv D_{i^{\prime \prime}}$ on figure 3.7. This new notion allows us to treat the disks on the boundary (the quasidisks) and the original disks uniformly. In particular, every vertex $x_{i}$ of the discrete network $\mathbb{D}$ is a center of a disk (or a quasidisk) and for a quasidisk the definition of a neighbor (definition 7) applies. 
Definition 21 The triangle-neck partition $\mathbb{P}=\mathbb{P}\left(Q_{p}\right)$ of the domain $Q_{p}$ is the set of necks $\Pi_{i j}$ and triangles $\Delta_{i j k}$.

The triangle-neck partition is unique up to partitioning of degenerate (exceptional) polygons into triangles. The necks $\Pi_{i j}$ connect two disks (in particular, a disk and a quasidisk) $D_{i}$ and $D_{j}$ if they are neighbors. All vertices of the triangles lie on the circumferences of the disks (or quasidisks).

Since from the physical point of view, for a discrete network $\mathbb{D}$ the necks of its $\delta$-subgraph carry the main information about the effective properties of the material, we wish to show that all the triangles and the "non-essential" (longer than $\delta$ ) necks do not contribute to the discrete network approximation. In order to do that, we need an estimate on the number of such non-essential necks and triangles in the triangle-neck partition relative to the number of essential (shorter than $\delta$ ) necks.

Remark 2 For a given distribution of disks there is the one-to-one correspondence between its discrete network $\mathbb{D}$ and the associated triangle-neck partition where the necks $\Pi i j$ are associated with the edges $e_{i j}$, and the triangles $\Delta_{i j k}$ are associated with the respective 3 -cycles of the graph $\mathbb{D}$. Therefore we will use instead of the edges $e_{i j}$ the corresponding necks $\Pi_{i j}$ where there is no confusion.

The next lemma gives an upper bound on the number of necks and triangles that lie in the interior of any minimal cycle of the $\delta$-subgraph in terms of the size of largest minimal cycle $\mathcal{N}$. Consider a $\delta$ - $\mathcal{N}$ connected discrete network $\mathbb{D}$ (definition 17). Consider a minimal cycle $C_{\mathrm{m}}$ of the $\delta$-subgraph $\mathbb{D}_{\delta}$. Denote by $\# \Delta_{C \mathrm{~m}}$ the number of the triangles $\Delta_{i j k}$ that lie in the interior of this minimal cycle $\Delta_{i j k} \subset \operatorname{Int}_{C \mathrm{~m}}$. Similarly $\# \Pi_{C \mathrm{~m}}$ is the number of the necks $\Pi_{i j}$ that lie in the interior of this minimal cycle $\Pi_{i j} \subset \operatorname{Int}_{C \mathrm{~m}}$, and $\# x_{C \mathrm{~m}}$ is the number of the vertices (centers of disks) $x_{i}$ such that $x_{i} \subset \operatorname{Int}_{C \mathrm{~m}}$.

Lemma 1 Suppose the discrete network $\mathbb{D}$ is $\delta$ - $\mathcal{N}$ connected. Then for any minimal cycle $C_{m}$ of the $\delta$-subgraph $\mathbb{D}_{\delta}$ the number of triangles $\# \Delta_{C m}$, and the number of necks $\# \Pi_{C m}$ that lie in the interior of $C_{m}$ satisfy the bounds

$$
\begin{aligned}
\# \Delta_{C_{m}} & \leq 2\left(\mathcal{N}+\frac{2}{\pi \sqrt{3}} \mathcal{N}^{2}\right), \\
\# \Pi_{C_{m}} & \leq 3\left(\mathcal{N}+\frac{2}{\pi \sqrt{3}} \mathcal{N}^{2}\right) .
\end{aligned}
$$

Proof: Consider a cycle $C_{\mathrm{m}}$. By Euler's formula

$$
\# \Delta_{C_{\mathrm{m}}}-\# \Pi_{C_{\mathrm{m}}}+\# x_{C_{\mathrm{m}}}=1 \text {. }
$$

Since each triangle has three edges and each edge belongs to at most two triangles

$$
3 \# \Delta_{C \mathrm{~m}} \leq 2 \# \Pi_{C_{\mathrm{m}}}
$$

Therefore

$$
\begin{aligned}
\# \Delta_{C_{\mathrm{m}}} \leq 2\left(\# \Pi_{C_{\mathrm{m}}}-\# \Delta_{C_{\mathrm{m}}}\right) & \leq 2 \# x_{C_{\mathrm{m}}} \\
\# \Pi_{C_{\mathrm{m}}} \leq x_{C_{\mathrm{m}}}+\# \Delta_{C_{\mathrm{m}}} & \leq 3 \# x_{C_{\mathrm{m}}} .
\end{aligned}
$$


If $C_{\mathrm{m}}$ is a minimal cycle of $\mathbb{D}_{\delta}$, then by $\delta$ - $\mathcal{N}$ connectedness the length of this cycle is less than $\mathcal{N}(2 R+\delta)$. By the isoperimetric inequality the area of the interior of an internal cycle is maximal, when it is a disk of the radius $\mathbf{R}_{0}=\mathbf{R}_{0}(\mathcal{N}, R, \delta)$; however, a boundary cycle with maximal area is a half-circle at the boundary. Therefore the radius of the half-circle which corresponds to the boundary cycle of length $N$ is greater than $\mathbf{R}_{0}$. and an upper bound on this latter area provides an upper bound on the area of the interior of any cycle

$$
\frac{\pi}{2}\left(\frac{\mathcal{N}(2 R+\delta)}{\pi}\right)^{2}=2 \frac{\mathcal{N}^{2}(R+\delta / 2)^{2}}{\pi} .
$$

Let us compute the maximal number of disks of radius $R$ that can be placed inside a half-disk with the perimeter $\mathcal{N}(2 R+\delta)$. Suppose this number is $K$. Since $C_{\mathrm{m}}$ is a minimal cycle each disk in the interior must be separated from any other disk by $\delta$. Therefore the total area covered by $K$ disks with the (densest possible) hexagonal packing is $2 \sqrt{3}(R+\delta / 2)^{2} K$. By our assumption that all centers of the disks lie inside the domain $\Pi$, at least a half of the disk lies in the interior of $C \mathrm{~m}$. Hence the total area covered by $K$ parts of disks with centers inside $\Pi$ is $\sqrt{3}(R+\delta / 2)^{2} K$. Therefore we have

$$
\sqrt{3}(R+\delta / 2)^{2} K \leq 2 \frac{\mathcal{N}^{2}(R+\delta / 2)^{2}}{\pi}
$$

So

$$
K \leq \frac{2}{\pi \sqrt{3}} \mathcal{N}^{2}
$$

The total number of vertices $x_{i} \in\{x\}_{C_{\mathrm{m}}}$ satisfies

$$
\# x_{C_{\mathrm{m}}} \leq K+\mathcal{N} \leq \mathcal{N}+\frac{2}{\pi \sqrt{3}} \mathcal{N}^{2}
$$

By (3.21)

$$
\begin{aligned}
& \# \Delta_{C_{\mathrm{m}}} \leq 2\left(\mathcal{N}+\frac{2}{\pi \sqrt{3}} \mathcal{N}^{2}\right) \\
& \# \Pi_{C_{\mathrm{m}}} \leq 3\left(\mathcal{N}+\frac{2}{\pi \sqrt{3}} \mathcal{N}^{2}\right)
\end{aligned}
$$

\subsection{The discrete minimization problem}

Here we describe the discrete minimization problem. In section 3.2 we have already described the discrete network (definition 5). In order to define the discrete minimization problem (1.5) we only need to determine the coefficients $g_{i j}^{0}$ and boundary conditions. In fact, in this section we describe two discrete minimization problems, which differ from each other by the choice of coefficients $g_{i j}^{0}$. In the $\mathbb{D}$-model these coefficients (denoted by $g_{i j}$ as in [4]) are determined solely by the parameters $\delta_{i j}$ of the discrete network $\mathbb{D}$ (definition 5). In the $\mathbb{D P}$-model the coefficients $g_{i j}^{0}$ are determined by $\delta_{i j}$ and the widths of necks $\Pi_{i j}$ of the triangle-neck partition $\mathbb{P}$ (definition 


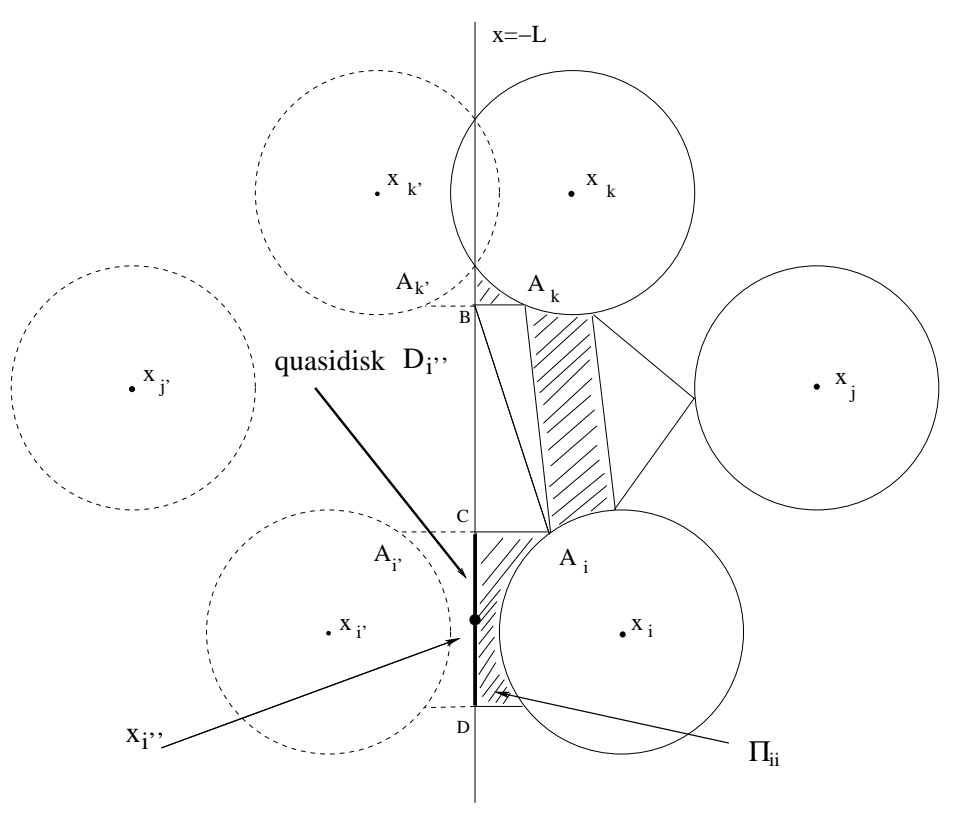

Figure 3.7: Vertical boundary

21). The $\mathbb{D}$-model is simpler, because it does not use the widths of necks $\Pi_{i j}$, but the error estimates for the $\mathbb{D P}$-model are better.

Let us begin here with the $\mathbb{D}$-model. For a given distribution of disks $D_{i}$ with centers $x_{i}, i=1, \ldots, N$ define the discrete network $\mathbb{D}$ with vertices $x_{i} i=1, \ldots, M$, $M \geq N$ and edges $e_{i j}$. For $\tilde{\mathbf{t}}=\left(\tilde{t}_{1}, \tilde{t}_{2}, \ldots, \tilde{t}_{M}\right)$ consider

$$
I(\tilde{\mathbf{t}})=\frac{1}{4 L} \sum_{\Pi_{i j}} g_{i j}\left(\tilde{t}_{i}-\tilde{t}_{j}\right)^{2} .
$$

Here the summation is over all necks $\Pi_{i j}$. The relative interparticle fluxes $g_{i j}$ are defined by (3.5). The discrete potentials $\tilde{t}_{i}$ at the "boundary" vertices $x_{i}$, are prescribed on the horizontal boundaries by

$$
\tilde{t}_{i}= \pm 1 \text { for } x_{i} \in S^{ \pm}
$$

where $S^{ \pm}$are the upper/lower boundary vertices (definition 6).

The discrete potentials $\tilde{t}_{i}$ at the "interior" vertices $x_{i} \in \mathbb{I} \mathbb{I}=\left\{x_{i}, i=1, \ldots, N\right\} \backslash$ $\left(S^{+} \cup S^{-}\right)$, are the values of discrete potentials. If a vertex of the discrete network $x_{i^{\prime \prime}} \notin \mathbb{I} \cup\left(S^{+} \cup S^{-}\right)$, then $x_{i^{\prime}}$ must be a center of a quasidisk, that lies on the left or the right boundary $x_{i^{\prime \prime}} \in S^{\mathrm{l}} \cup S^{\mathrm{r}}$. For such vertices we assume

$$
\tilde{t}_{i^{\prime \prime}}=\tilde{t}_{i}
$$


where $t_{i}$ is the value of the potential on the disk $D_{i} \in \mathbb{I} \cup\left(S^{+} \cup S^{-}\right), t_{i^{\prime \prime}}$ is the value of the potential on the quasidisk $D_{i^{\prime \prime}}$ connected with the disk $D_{i}$ (on figure 3.7 $D_{i^{\prime \prime}} \equiv C D$ ).

The discrete minimization problem is to find the potentials at the interior vertices, that is a set of $\mathbf{t}=\left\{t_{i} \mid x_{i} \in \mathbb{I}\right\}$, such that the quadratic form (3.22) with conditions (3.23),(3.24) is minimal, that is

$$
\begin{aligned}
& \min _{\tilde{t}_{i}, x_{i} \in \mathbb{I}} I(\tilde{\mathbf{t}})=I(\mathbf{t}) . \\
& (3.23),(3.24)
\end{aligned}
$$

Definition 22 If $\mathbf{t}=\left\{t_{i} \mid x_{i} \in \mathbb{I}\right\}$ is the solution of the discrete minimization problem (3.25), then

$$
I(\mathbf{t})=\frac{1}{4 L} \sum_{\Pi_{i j}} g_{i j}\left(t_{i}-t_{j}\right)^{2}
$$

is the energy of the discrete network for the $\mathbb{D}$-model.

The discrete network is a connected graph in the sense that there is a path between each vertex $x_{i}$ and a boundary vertex $x_{j} \in S^{ \pm}$. This implies that the discrete minimization problem has a unique solution, because if a graph is connected, then any local minimizer of the quadratic form (3.22) is the (unique) global minimizer. Therefore we have

Lemma 2 There is a unique solution $\mathbf{t}=\left\{t_{i} \mid x_{i} \in \mathbb{I}\right\}$ of the discrete minimization problem (3.25). This solution satisfies a discrete analog of Euler-Lagrange equations (compare to (2.5))

$$
\sum_{\Pi_{i k}, i \text { fixed }} g_{i k}\left(t_{i}-t_{k}\right)=0 \text { for all } x_{i} \in \mathbb{I} .
$$

Since the quadratic form (3.22) is positive-definite the proof follows immediately from linear algebra.

Remark 3 The summation in (3.27) is over necks $\Pi_{i k}$, such that one of their endpoints $x_{i}$ is fixed. All these necks must be incident to the vertex $x_{i}$. In the sequel we will use the same notation.

The values $t_{i}^{\prime \prime}$, for $x_{i}^{\prime \prime} \notin \mathbb{I} \cup\left(S^{+} \cup S^{-}\right)$play no role in the minimization process and can be determined a posteriori by (3.24). In particular we will need the following

$$
\sum_{\Pi_{i k}, i^{\prime \prime}} g_{i^{\prime \prime} k}\left(t_{i^{\prime \prime}}-t_{k}\right)=g_{i^{\prime \prime} i}\left(t_{i^{\prime \prime}}-t_{i}\right)=0 \text { for all } x_{i} \notin \mathbb{I} \cup\left(S^{+} \cup S^{-}\right) .
$$

Denote by $P^{+}$and $P^{-}$the discrete fluxes (confer to (2.6)) through the boundaries $S^{+}$and $S^{-}$respectively

$$
\begin{aligned}
P^{+} & \equiv \sum_{\Pi_{i j}, x_{i} \in S^{+}} g_{i j}\left(t_{i}-t_{j}\right) \\
P^{-} & \equiv \sum_{\Pi_{i j}, x_{i} \in S^{-}} g_{i j}\left(t_{i}-t_{j}\right) .
\end{aligned}
$$


Then

$$
P^{+}=-P^{-}=\frac{1}{2} \sum_{\Pi_{i j}} g_{i j}\left(t_{i}-t_{j}\right)^{2} \equiv 2 L I(\mathbf{t}),
$$

where the first and the second equalities are the discrete analogs of formulas (2.6) and (2.12) respectively. The derivation of (3.30) uses (3.27) and the following rearrangement of terms. The first equality $P^{+}=-P^{-}$holds, because

$$
\begin{aligned}
& P^{+}=\sum_{\Pi_{i j}, x_{i} \in S^{+}} g_{i j}\left(t_{i}-t_{j}\right)=\sum_{\Pi_{i j}, x_{i} \in S^{+}} g_{i j}\left(t_{i}-t_{j}\right)+\sum_{\Pi_{i j}, x_{i} \notin S^{ \pm}} g_{i j}\left(t_{i}-t_{j}\right) \\
& =\sum_{\Pi_{i j}, x_{j} \notin S^{ \pm}} g_{i j}\left(t_{i}-t_{j}\right)+\sum_{\Pi_{i j}, x_{j} \in S^{-}} g_{i j}\left(t_{i}-t_{j}\right)=\sum_{\Pi_{i j}, x_{j} \in S^{-}} g_{i j}\left(t_{i}-t_{j}\right)=-P^{-} .
\end{aligned}
$$

The second equality in (3.30) can be deduced from (3.27) and (3.28)

$$
\begin{aligned}
& 4 L I(\mathbf{t})=\sum_{\Pi_{i j}} g_{i j}\left(t_{i}-t_{j}\right)^{2}=\sum_{\Pi_{i j}} g_{i j}\left(t_{i}-t_{j}\right)\left(t_{i}-t_{j}\right) \\
& =\sum_{x_{i}} t_{i}\left[\sum_{\Pi_{i j}, i \text { fixed }} g_{i j}\left(t_{i}-t_{j}\right)\right]+\sum_{x_{j}} t_{j}\left[\sum_{\Pi_{i j}, j \text { fixed }} g_{j i}\left(t_{j}-t_{i}\right)\right] \\
& =\sum_{x_{i} \in \mathbb{I} \cup\left(S+\cup S^{-}\right)} t_{i}\left[\sum_{\Pi_{i j}, i \text { fixed }} g_{i j}\left(t_{i}-t_{j}\right)\right]+\sum_{x_{j} \in \mathbb{I} \cup\left(S^{+} \cup S^{-}\right)} t_{j}\left[\sum_{\Pi_{i j}, j \text { fixed }} g_{j i}\left(t_{j}-t_{i}\right)\right] \\
& =\sum_{\Pi_{i j}, x_{i} \in S^{+}} g_{i j}\left(t_{i}-t_{j}\right)+\sum_{\Pi_{i j}, x_{i} \in S^{-}} g_{i j}\left(t_{j}-t_{i}\right)=P^{+}-P^{-} .
\end{aligned}
$$

Lemma 3 Discrete maximum principle. Suppose $\mathbf{t}=\left\{t_{1}, t_{2}, \ldots t_{M}\right\}$ is the solution of the $\mathbb{D}$ problem (3.25). For any (internal or boundary) cycle $C$ of $\mathbb{D}$ define

$$
t_{\max }=\max \left(t_{i}\right), x_{i} \in C, \quad t_{\min }=\min \left(t_{i}\right), x_{i} \in C .
$$

Then for any vertex $x_{k}$ with potential $t_{k}$ such that $x_{k} \in$ Int $_{C}$, that is $x_{k}$ belongs to the interior of the cycle $C$ (see definition 16) we have

$$
t_{\min } \leq t_{k} \leq t_{\max }
$$

Proof: The proof of the discrete maximum principle is by contradiction, and it is fairly standard. Suppose not all the values $t_{i}$ for the points $x_{i}$ in the interior of the cycle are the same and the maximum of $t_{i}$ is achieved in the proper interior of the cycle at a point $x_{\mathbf{k}} \in \operatorname{Int}_{C}, x_{k} \notin C$. Then we have $x_{i} \leq x_{\mathbf{k}}$ for any $x_{i} \in \operatorname{Int}_{C}$. Therefore, the discrete Euler-Lagrange equation (3.27) is satisfied only if $t_{i} \equiv t_{\mathbf{k}}$ whenever vertices $x_{i}$ and $x_{\mathbf{k}}$ are connected by a neck $\Pi_{i \mathbf{k}}$. Hence the maximum is also achieved at all the points $x_{i}$ which are connected with $x_{\mathbf{k}}$ by a neck $\Pi_{i \mathbf{k}}$. Since the graph is connected, the induction over all the points in the interior of the cycle implies the contradiction: the values $t_{i}$ for all the points in the interior of the cycle are the same.

As a corollary of the discrete maximum principle we have 
Lemma 4 If the discrete network $\mathbb{D}$ is $\delta$ - $\mathcal{N}$ connected, then for any minimal cycle $C$ of the $\delta$-subgraph $\mathbb{D}_{\delta}$ and a vertices $x_{k} \in$ Int $_{C}$ and $x_{l} \in$ Int $_{C}$ we have

$$
\left(t_{k}-t_{l}\right)^{2} \leq \mathcal{N} \sum_{\Pi_{i j} \in C m}\left(t_{i}-t_{j}\right)^{2}
$$

Proof: By the discrete maximum principle (lemma 3)

$$
\left(t_{k}-t_{l}\right)^{2} \leq\left(t_{\max }-t_{\min }\right)^{2}
$$

where

$$
t_{\max }=\max \left(t_{i}\right), x_{i} \in C, \quad t_{\min }=\min \left(t_{i}\right), x_{i} \in C .
$$

By the triangle inequality for the values of the potentials $t_{i}, x_{i} \in C$

$$
\left(t_{\max }-t_{\min }\right)^{2} \leq \mathcal{N} \sum_{\Pi_{i j} \in C_{\mathrm{m}}}\left(t_{i}-t_{j}\right)^{2}
$$

which inserted in (3.32) yields (4).

Let us discuss now the $\mathbb{D P}$-model. For a given distribution of disks $D_{i} x_{i}, i=$ $1, \ldots, N$ define the discrete network $\mathbb{D}$ with vertices $x_{i} i=1, \ldots, M, M \geq N$ and edges $e_{i j}$ and the triangle-neck partition $\mathbb{P}$ (definition 21). Then the specific fluxes $g_{i j}^{0}$ can be determined by formula (3.4) in terms of necks $\Pi_{i j}$. Then, similar to the first model, we define

$$
I_{0}(\tilde{\mathbf{t}})=\frac{1}{4 L} \sum_{\Pi_{i j}} g_{i j}^{0}\left(\tilde{t}_{i}-\tilde{t}_{j}\right)^{2}
$$

and the second discrete minimization problem: find a set $\mathbf{t}=\left\{t_{i} \mid x_{i} \in \mathbb{I}\right\}$, such that

$$
\min _{\tilde{t}_{i}, x_{i} \in \mathbb{I}} \quad I_{0}(\tilde{\mathbf{t}})=I_{0}\left(\mathbf{t}^{\mathbf{0}}\right),
$$

here the energy of the discrete network for the $\mathbb{D P}$-model

$$
I_{0}\left(\mathbf{t}^{\mathbf{0}}\right)=\frac{1}{4 L} \sum_{\Pi_{i j}} g_{i j}^{0}\left(t_{i}^{0}-t_{j}^{0}\right)^{2} .
$$

For the second discrete minimization problem one can derive properties similar to lemmas 2, 3, equation (3.27) etc. Quantities $P_{0}^{ \pm}$similar to $P^{ \pm}$can be defined as well. Let us compare the two discrete minimization problems for quadratic forms

$$
I(\mathbf{t})=\frac{1}{4 L} \sum_{\Pi_{i j}} g_{i j}\left(t_{i}-t_{j}\right)^{2}
$$

and

$$
I_{0}(\mathbf{t})=\frac{1}{4 L} \sum_{\Pi_{i j}} g_{i j}^{0}\left(t_{i}-t_{j}\right)^{2}
$$


By (A.17)

$$
g_{i j}^{0}<g_{i j}
$$

therefore for any $\tilde{\mathbf{t}}=\left\{\tilde{t}_{1}, \tilde{t}_{2}, \ldots \tilde{t}_{M}\right\}$ we have

$$
I_{0}(\tilde{\mathbf{t}})<I(\tilde{\mathbf{t}})
$$

Hence

$$
\begin{array}{ll}
\min _{\tilde{t}_{i}, x_{i} \in \mathbb{I}} & I_{0}(\tilde{\mathbf{t}})< \\
(3.23),(3.24) & \min _{\tilde{t}_{i}, x_{i} \in \mathbb{I}} I(\tilde{\mathbf{t}}) . \\
& (3.23),(3.24)
\end{array}
$$

Suppose $\min I_{0}(\mathbf{t})$ and $\min I(\mathbf{t})$ is achieved on the sets $\mathbf{t}^{0}=\left\{t_{1}^{0}, t_{2}^{0}, \ldots t_{M}^{0}\right\}$ and $\mathbf{t}=\left\{t_{1}, t_{2}, \ldots t_{M}\right\}$ respectively. Then

$$
\begin{aligned}
& I(\mathbf{t})=\frac{1}{4 L} \sum_{\Pi_{i j}} g_{i j}\left(t_{i}-t_{j}\right)^{2} \leq \frac{1}{4 L} \sum_{\Pi_{i j}} g_{i j}\left(t_{i}^{0}-t_{j}^{0}\right)^{2}=\frac{1}{4 L} \sum_{\Pi_{i j}} g_{i j}^{0}\left(t_{i}^{0}-t_{j}^{0}\right)^{2} \\
& +\frac{1}{4 L} \sum_{\Pi_{i j}}\left(g_{i j}-g_{i j}^{0}\right)\left(t_{i}^{0}-t_{j}^{0}\right)^{2}=I_{0}\left(\mathbf{t}^{0}\right)+\frac{1}{4 L} \sum_{\Pi_{i j}}\left(g_{i j}-g_{i j}^{0}\right)\left(t_{i}^{0}-t_{j}^{0}\right)^{2} .
\end{aligned}
$$

Hence

$$
I_{0}\left(\mathbf{t}^{\mathbf{0}}\right)<I(\mathbf{t}) \leq I_{0}\left(\mathbf{t}^{\mathbf{0}}\right)+\frac{1}{4 L} \sum_{\Pi_{i j}}\left|g_{i j}-g_{i j}^{0}\right|\left(t_{i}^{0}-t_{j}^{0}\right)^{2} .
$$

In the cases we consider here $(\delta-\mathcal{N}$ connectedness, definition 17) we show in section 4 a tighter inequality

$$
I_{0}\left(\mathbf{t}^{\mathbf{0}}\right)<I(\mathbf{t}) \leq\left(1+C \sqrt{\frac{\delta}{R}}\right) I_{0}\left(\mathbf{t}^{\mathbf{0}}\right)
$$

\section{Variational bounds on the effective conductivity}

The classical approach to variational bounds is to find some "good" trial functions $\phi \in V_{p}$ and $\mathbf{v} \in W_{p}$ and apply inequality (2.16). The main difficulty is to find the trial functions. A successful choice of the trial functions leads to tight bounds. The idea of constructing tight bounds by means of variational principles is not new and have been used by many authors but each time the main question is the actual construction of dual test functions which is usually highly nontrivial and it relies on specific features of the problem. For example, the test functions for the dual bounds from [8] or [16] are very different from ours and from each other due to different geometries. There is no general recipe for choosing the trial functions. In this section we show that for our specific problem of effective conductivity of high contrast, highly packed particular composites we can construct the upper and the lower bounds for a composite when its discrete network $\mathbb{D}$ is $\delta$ - $\mathcal{N}$ connected (definition 17) and we estimate the difference between the upper and the lower bounds in terms of $\delta, \mathcal{N}$ and the energies $I(\mathbf{t})$ and $I\left(\mathbf{t}^{0}\right)$ of the discrete minimization problems (equations (3.26) and (3.35)). This allows to give an estimate on the relative error between the effective conductivity (2.9) and the 
energy of the discrete network (3.26). Certainly our trial functions give upper and lower variational bounds on effective conductivity for any distribution of disks; however, our bounds are tight only under the assumption that "almost all" the distances $\delta_{i j}$ between neighbors are sufficiently small ( $\delta$ - $\mathcal{N}$ connectedness).

Definition 23 A distribution of disks $D_{i}, i=1, \ldots, N$ satisfies the $\delta$ - $\mathcal{N}$ close packing condition if its discrete network $\mathbb{D}$ is $\delta$ - $\mathcal{N}$ connected.

We also give a better estimate on the relative error between the effective conductivity (2.9) and the energy of the discrete networks (3.26), (3.35) in two special cases: $\mathcal{N}=3$ and $\mathcal{N}=4$. If $\mathcal{N}=3$, then all neighbors are $\delta$-close to each other. The case $\mathcal{N}=3$ covers distributions of disks which are small perturbations of the periodic hexagonal lattice (figure 3.2), therefore it is important for applications. If $\mathcal{N}=4$ then the condition that all neighbors are $\delta$-close is relaxed. In particular, this case includes the periodic square lattice (figure 3.6).

Here is the the idea of the construction of the variational bounds. Our goal is to construct two trial functions, which mimic the behavior of the solution to the minimization problem (3.34) for the $\mathbb{D P}$-model. The domain $\Pi$ consists of disks $D_{i}$, necks $\Pi_{i j}$ and triangles $\Delta_{i j k}$ where the necks and triangles are determined by the triangleneck partition. The function $\phi \in V_{p}$ and the function $\mathbf{v} \in W_{p}$ are defined first on the disks, then in the necks and the triangles. Suppose $t_{i}^{0}$ are the values of the solution of the minimization problem (3.34) for the $\mathbb{D P}$-model. The function $\phi \in V_{p}$ is chosen so that it takes the values $t_{i}^{0}$ on the disks $D_{i}$, it is equal to \pm 1 on the boundaries $S^{ \pm}$ and it is linear in the necks. Observe that the formulation of the problem is rotationally invariant, therefore in the construction of the trial functions we routinely locally rotate the plane so that in the local coordinates a particular neck $\Pi_{i j}$ is always aligned with the vertical direction. The function $\phi$ should be piecewise-differentiable by definition of the space $V_{p}$ (2.2). Therefore on triangles it is defined by linear interpolation. The function $\mathbf{v} \in W_{p}$ intuitively is the flux of $\phi$, and it is chosen on disks and necks taking into account the discrete fluxes of the discrete minimization problem. The function $\mathbf{v}$ does not have to be piecewise-differentiable, hence we simply define it to be zero on the triangles.

The idea of the error estimate is to show that both the upper and lower bound are very close to the energy of the $\mathbb{D P}$-model. In order to show that the energy of the $\mathbb{D}$ model (3.25) is a good approximation as well we compute the difference between the solution of the $\mathbb{D P}$-model and the solution of the $\mathbb{D}$-model.

\subsection{The lower bound}

The test function $\mathbf{v}$ for the lower bound is chosen to be zero everywhere except the necks $\Pi_{i j}$ between adjacent disks. Consider two neighbors centered at $x_{i}$ and $x_{j}$. The potentials $t_{i}^{0}$ and $t_{j}^{0}$ on the disks $D_{i}$ and $D_{j}$ are the solutions to the minimization problem (3.34) for the $\mathbb{D P}$-model. Rotate the domain so that in the local coordinates the neck between them is aligned with the direction of the $y$-axis. Define

$$
\mathbf{v}= \begin{cases}\left(0, \frac{t_{i}^{0}-t_{j}^{0}}{H(x)}\right) & \text { in the neck } \Pi_{i j} \\ (0,0) & \text { otherwise }\end{cases}
$$


where $H(x)$ is the distance between the disks (figure 3.1). Let us check that the test function $\mathbf{v}$ lies in the space $W_{p}$ defined by (2.13). If at $\mathbf{x} \in \Pi_{i j}$ then divergence-free condition amounts to

$$
\nabla \cdot \mathbf{v}(\mathbf{x})=\frac{\partial}{\partial x} 0+\left(t_{i}^{0}-t_{j}^{0}\right) \frac{\partial}{\partial y} \frac{1}{H(x)}=0 .
$$

If $\mathbf{x} \in \Delta_{i j k}$ (see figure 4.2), then

$$
\nabla \cdot \mathbf{v}(\mathbf{x})=\frac{\partial}{\partial x} 0+\frac{\partial}{\partial y} 0=0 .
$$

If a point $\mathbf{x}$ lies on the boundary of the triangle-neck triangulation, that is $\mathbf{x} \in \partial \Pi_{i j} \cap$ $\partial \Delta_{i j}$, the vector field $\mathbf{v}$ is discontinuous there. In this case the divergence-free condition amounts to checking that the normal components of $\mathbf{v}$ match along the discontinuity. This matching is satisfied trivially for $\mathbf{v}$. The condition

$$
\int_{\partial D_{i}} \mathbf{v} \cdot \mathbf{n} d \mathbf{x}=0
$$

is satisfied due to (3.4). Indeed, we have

$$
\int_{\partial D_{i}} \mathbf{v} \cdot \mathbf{n} d \mathbf{x}=\sum_{j, \Pi_{i j}}\left(t_{i}^{0}-t_{j}^{0}\right) \int_{S_{1}}^{S_{2}} \frac{d x}{H(x)}=\sum_{j, \Pi_{i j}} g_{i j}^{0}\left(t_{i}^{0}-t_{j}^{0}\right)=0 .
$$

Here the last sum is zero for any $i \in \mathbb{I}$ by (3.27). By the quasidisk construction (3.28) the condition

$$
\mathbf{v}( \pm L, y) \cdot \mathbf{n}=0
$$

is also satisfied. Observe that for the trial function (4.1) the fluxes through the upper and the lower boundary of $\Pi$ are exactly the discrete fluxes $P_{0}^{+}$and $P_{0}^{-}$for the $\mathbb{D P}$ model

$$
\begin{gathered}
\int_{y=1} \mathbf{v} \cdot \mathbf{n} d \mathbf{x}=\sum_{x_{i} \in S^{+}} g_{i j}^{0}\left(t_{i}^{0}-t_{j}^{0}\right)=P_{0}^{+} \\
\int_{y=-1} \mathbf{v} \cdot \mathbf{n} d \mathbf{x}=\sum_{x_{i} \in S^{-}} g_{i j}^{0}\left(t_{i}^{0}-t_{j}^{0}\right)=P_{0}^{-} .
\end{gathered}
$$

Therefore similar to (3.30)

$$
\int_{y=1} \mathbf{v} \cdot \mathbf{n} d \mathbf{x}-\int_{y=-1} \mathbf{v} \cdot \mathbf{n} d \mathbf{x}=P_{0}^{+}-P_{0}^{-}=\sum_{\Pi_{i j}} g_{i j}^{0}\left(t_{i}^{0}-t_{j}^{0}\right)^{2}
$$

The value of the integral over the neck $\Pi_{i j}$ is

$$
\int_{\Pi_{i j}} \mathbf{v}^{2} d \mathbf{x}=\left(t_{i}^{0}-t_{j}^{0}\right)^{2} \int_{S_{1}}^{S_{2}} \frac{d x}{H(x)}=g_{i j}^{0}\left(t_{i}^{0}-t_{j}^{0}\right)^{2}
$$

and

$$
\int_{Q_{p}} \mathbf{v}^{2} d \mathbf{x}=\sum_{\Pi_{i j}} g_{i j}^{0}\left(t_{i}^{0}-t_{j}^{0}\right)^{2}
$$


By (4.2) and (4.3)

$$
\int_{y=1} \mathbf{v} \cdot \mathbf{n} d \mathbf{x}-\int_{y=-1} \mathbf{v} \cdot \mathbf{n} d \mathbf{x}-\frac{1}{2} \int_{Q_{p}} \mathbf{v}^{2} d \mathbf{x}=\frac{1}{2} \sum_{\Pi_{i j}} g_{i j}^{0}\left(t_{i}^{0}-t_{j}^{0}\right)^{2} .
$$

Hence we have a lower bound

Proposition 4.1 The lower bound on $\widehat{a}$ in terms of $g_{i j}^{0}$ and the parameters of the solution of the discrete minimization problem is

$$
I_{0}\left(\mathbf{t}^{\mathbf{0}}\right)=\frac{1}{4 L} \sum_{\Pi_{i j}} g_{i j}^{0}\left(t_{i}^{0}-t_{j}^{0}\right)^{2} \leq \widehat{a} .
$$

We remark here that the left-hand side in (4.1) is always positive, which reflects the physics of the problem. The analogous lower bound in Proposition 2.1 in [4] is positive and it is sufficiently tight for the $\delta$-3 close packing condition only. Our bound allows us to handle general distribution of disks that satisfy $\delta$ - $\mathcal{N}$ close packing condition for any $\mathcal{N}$.

\subsection{The upper bound}

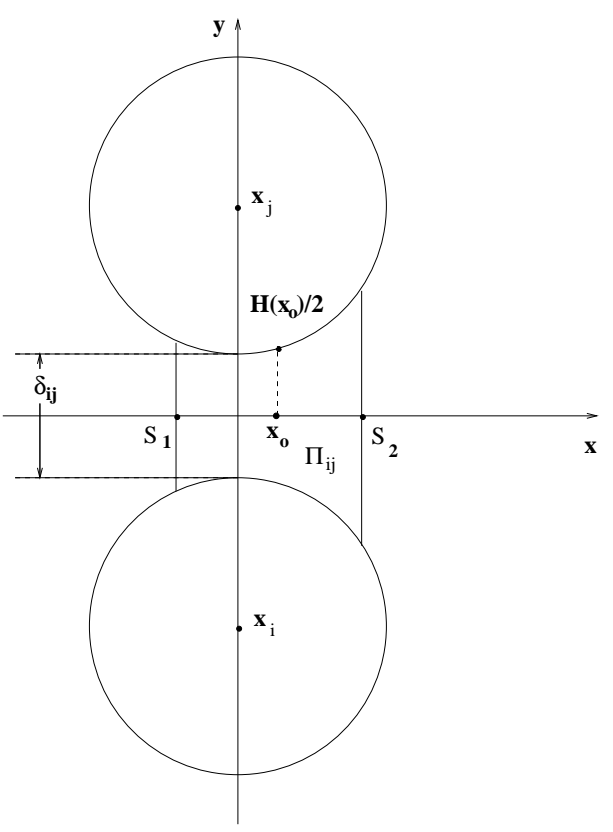

Figure 4.1: A typical neck between two disks. 


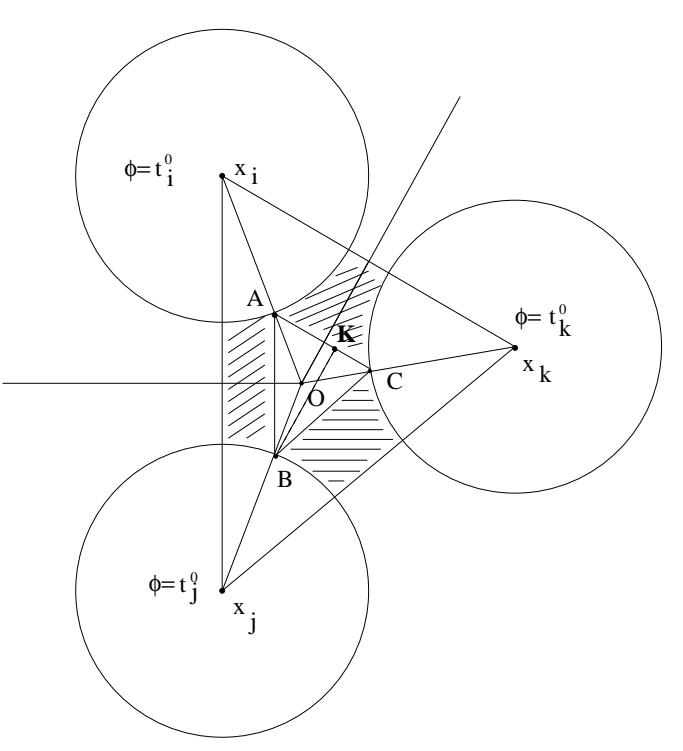

Figure 4.2: Typical $\triangle A B C \equiv \Delta_{i j k}$, half-necks are hatched.

Consider a piecewise continuous test function $\phi$. The function $\phi$ is linear in $y$ in the neck $\Pi_{i j}$ with the values $t_{i}^{0}$ and $t_{j}^{0}$ on the boundary of the disks $\partial D_{i}$ and $\partial D_{j}$ (figure 4.1). Then on the neck $\Pi_{i j}$

$$
\begin{aligned}
& \phi(x, y)=t_{i}^{0}+\frac{\left(t_{j}^{0}-t_{i}^{0}\right)(y+H(x) / 2)}{H(x)}=t_{i}^{0}+\left(t_{j}^{0}-t_{i}^{0}\right)\left[\frac{y}{H(x)}+\frac{1}{2}\right], \\
& \text { for } y \in\left[-\frac{H(x)}{2}, \frac{H(x)}{2}\right] .
\end{aligned}
$$

The function $\phi$ is linear in the $\Delta_{i j k}$ (see figure 4.2) with the values $t_{i}^{0}, t_{j}^{0}$ and $t_{k}^{0}$ at the vertices of $\Delta_{i j k}$. On figure 4.2 these vertices are the points $A, B$ and $C$ respectively.

The estimates on the $\int_{Q_{p}}|\nabla \phi|^{2} d \mathbf{x}$ depend on the bounds on the relative half-neck widths $\beta_{i j}^{\max }$ ( see (3.17)) for closely spaced neighbors $D_{i}$ and $D_{j}$. Such estimates are derived in appendix A.1 for the $\delta$ - $\mathcal{N}$ closely packed disks. These estimates are uniform for fixed $\delta$ and $\mathcal{N}$, that is

$$
\beta_{i j}^{\max } \leq \beta<1
$$

where $\beta$ is a number independent of $i$ and $j$, computed in appendix A.1. Assumption (4.6) for all practical cases usually satisfied with much better estimates than our worst case scenario estimates in (A.2)-(A.4) (lemma 6). By (A.19) and (A.11) and (4.6) we have 
Proposition 4.2 The upper bound on $\widehat{a}$ in terms of $g_{i j}^{0}$ and the parameters of the solution of the discrete minimization problem is

$$
\begin{aligned}
\widehat{a} & \leq \frac{1}{4 L} \sum_{\Pi_{i j}}\left[g_{i j}^{0}+\frac{|\ln (1-\beta)|+\pi+\ln 2}{6}\right]\left(t_{i}^{0}-t_{j}^{0}\right)^{2} \\
& +\frac{1}{4 L} \frac{2}{\sqrt{1-\beta^{2}}} \sum_{\Delta_{i j k}, \beta_{j k}^{\max }=\max \left(\beta_{i j}^{\max }, \beta_{k j}^{\max }, \beta_{k i}^{\max }\right)}\left(\left(t_{i}^{0}-t_{j}^{0}\right)^{2}+\left(t_{k}^{0}-t_{i}^{0}\right)^{2}\right) .
\end{aligned}
$$

\subsection{The error estimate}

As we already mentioned, the error of approximation of the minimization problem of effective conductivity (2.9) by the energy of $\mathbb{D P}$-model (3.25) is based solely on propositions 4.1 and 4.2, where if we assume the non-degenerate case (that is all the triangles $\Delta_{i j k}$ are adjacent to three necks $\Pi_{i j}, \Pi_{k j}$ and $\Pi_{k i}$ ), then we have

$$
\begin{aligned}
& \frac{1}{4 L} \sum_{\Pi_{i j}} g_{i j}^{0}\left(t_{i}^{0}-t_{j}^{0}\right)^{2} \leq \widehat{a} \leq \frac{1}{4 L} \sum_{\Pi_{i j}}\left[g_{i j}^{0}+\text { Const }\right]\left(t_{i}^{0}-t_{j}^{0}\right)^{2}, \\
& \text { where Const }=\frac{|\ln (1-\beta)|+\pi+\ln 2}{6}+\frac{4}{\sqrt{1-\beta^{2}}}=O(1) \text { as } \delta \rightarrow 0 .
\end{aligned}
$$

Equation (4.8) simply reflects the fact that the effective conductivity $\widehat{a}$ is the sum of the integrals in the $y$-direction over all necks (here we use our local coordinate systems), the sum of the integrals in the $x$-direction over all necks and the sum of the integrals over all triangles. The sum of the integrals in the $y$-direction over all necks is equivalent to the energy $I$ of the $\mathbb{D}$-model (3.26) and therefore it is $O\left(\sqrt{\frac{R}{\delta}}\right)$ as $\delta \rightarrow 0$. The sum of the integrals in the $x$-direction over all necks and the sum of the integrals over all triangles is $O(1)$ as $\delta \rightarrow 0$ (see appendices A.2 and A.4 respectively). Similar to (4.8) we have an error estimate between the energies of the $\mathbb{D P}$-model and the $\mathbb{D}$-model

$$
\begin{aligned}
& \frac{1}{4 L} \sum_{\Pi_{i j}} g_{i j}^{0}\left(t_{i}^{0}-t_{j}^{0}\right)^{2} \leq \frac{1}{4 L} \sum_{\Pi_{i j}} g_{i j}\left(t_{i}-t_{j}\right)^{2} \\
& \leq \frac{1}{4 L} \sum_{\Pi_{i j}} g_{i j}^{0}\left(t_{i}^{0}-t_{j}^{0}\right)^{2}+\frac{1}{4 L} \sum_{\Pi_{i j}}\left|g_{i j}-g_{i j}^{0}\right|\left(t_{i}^{0}-t_{j}^{0}\right)^{2},
\end{aligned}
$$

where $\left|g_{i j}-g_{i j}^{0}\right|=O(1)$ by (A.17).

Hence the idea of the proof: we wish to "absorb" all the $O(1)$ terms in (4.8) and (4.9) as the "smaller order corrections" into $O\left(\sqrt{\frac{R}{\delta}}\right)$ the terms and derive an estimate

$$
I \leq \widehat{a} \leq I+O(1) \leq I\left(1+C \sqrt{\frac{\delta}{R}}\right) .
$$

Then we have

$$
\frac{|\widehat{a}-I|}{I} \leq \mathbf{C} \sqrt{\frac{\delta}{R}}
$$


The main goal of the theorems in this section is to evaluate $\mathbf{C}$ in (4.10).

Theorem 4.3 Under the $\delta$ - $\mathcal{N}$ close packing condition (definition 23) for $\delta \leq R / 32$ the relative error

$$
\frac{\left|\widehat{a}-I_{0}\right|}{I_{0}} \leq 2.56 \mathcal{N}^{4} \sqrt{\frac{\delta}{R}}
$$

where the effective conductivity

$$
\begin{aligned}
& \widehat{a}=\frac{1}{4 L} \int_{Q_{p}}|\nabla \phi|^{2} d x \\
& \frac{1}{4 L} \int_{Q_{p}}|\nabla \phi|^{2} d x=\frac{1}{4 L} \min _{\tilde{\phi} \in V_{p}} \int_{Q_{p}}|\nabla \tilde{\phi}|^{2} d x
\end{aligned}
$$

and the energy of the $\mathbb{D P}$-model

$$
\begin{aligned}
& I_{0} \equiv I_{0}(\mathbf{t})=\frac{1}{4 L} \sum_{\Pi_{i j}} g_{i j}^{0}\left(t_{i}^{0}-t_{j}^{0}\right)^{2}, \\
& \frac{1}{4 L} \sum_{\Pi_{i j}} g_{i j}^{0}\left(t_{i}^{0}-t_{j}^{0}\right)^{2}=\frac{1}{4 L} \min _{\tilde{\mathbf{t}}^{0}=\left\{\tilde{t}_{1}^{0}, \tilde{t}_{2}^{0}, \ldots \tilde{t}_{M}^{0}\right\}} \sum_{\Pi_{i j}} g_{i j}^{0}\left(\tilde{t}_{i}^{0}-\tilde{t}_{j}^{0}\right)^{2} .
\end{aligned}
$$

Remark 4 The factor $1 / 32$ in theorem 4.3 is chosen for the simplicity of presentation and it is not essential. If our estimates on $\left|g_{i j}-g_{i j}^{0}\right|$ in appendix $A .3$ and the proof of theorem 4.3 are modified, the statement of theorem 4.3 is true for any $\delta \leq R$.

Proof: By proposition 4.1, the effective conductivity $\widehat{a}$ is bounded from below

$$
I_{0}\left(\mathbf{t}^{\mathbf{0}}\right)=\frac{1}{4 L} \sum_{\Pi_{i j}} g_{i j}^{0}\left(t_{i}^{0}-t_{j}^{0}\right)^{2} \leq \widehat{a} .
$$

By proposition 4.2, the effective conductivity $\widehat{a}$ is bounded from above

$$
\widehat{a} \leq I_{0}\left(\mathbf{t}^{0}\right)+C \sum_{\Pi_{i j}}\left(t_{i}^{0}-t_{j}^{0}\right)^{2}+C \sum_{\Delta_{i j k}}\left(t_{i}^{0}-t_{j}^{0}\right)^{2}
$$

Where the last two summations in (4.12) are respectively over all neck and triangles of the triangle-neck partition. Our goal is to bound (4.12) by the following sum

$$
\frac{1}{4 L} \sum_{\Pi_{i j} \in \mathbb{D}_{\delta}}\left(g_{i j}^{0}+\mathbf{C}\right)\left(t_{i}^{0}-t_{j}^{0}\right)^{2}+\frac{1}{4 L} \sum_{\Pi_{i j} \notin \mathbb{D}_{\delta}} g_{i j}^{0}\left(t_{i}^{0}-t_{j}^{0}\right)^{2}
$$

where $\mathbb{D}_{\delta}$ is the $\delta$-subgraph of the discrete network $\mathbb{D}$ (definition 9). Since

$$
g_{i j}^{0}+C=g_{i j}^{0}\left(1+C \sqrt{\frac{\delta}{R}}\right), \text { when } \Pi_{i j} \in \mathbb{D}_{\delta}
$$

we obtain (4.11). The basic reason for deducing (4.13) from (4.12) is that the key relation (4.14) is guaranteed only for the necks of the $\delta$-subgraph $\mathbb{D}_{\delta}$. Full graph $\mathbb{D}$ may contain "long" necks, for which (4.14) does not hold.

The proof of the theorem consists of three basic estimates 
- For any minimal cycle $C_{\mathrm{m}}$ recall the notation

- \# $\Delta_{C \mathrm{~m}}$ is the number of triangles $\Delta_{i j k}$, such that $\Delta_{i j k} \subset \operatorname{Int}_{C \mathrm{~m}}$,

- $\# \Pi_{C \mathrm{~m}}$ is the number of necks, $\Pi i j$ such that $\Pi_{i j} \subset \operatorname{Int}_{C \mathrm{~m}}$,

- \# $x_{C \mathrm{~m}}$ is the number of vertices $x_{i}$, such that $x_{i} \subset \operatorname{Int}_{C \mathrm{~m}}$.

By lemma 1

$$
\begin{aligned}
& \# \Delta_{C_{\mathrm{m}}} \leq 2\left(\mathcal{N}+\frac{2}{\pi \sqrt{3}} \mathcal{N}^{2}\right) \\
& \# \Pi_{C_{\mathrm{m}}} \leq 3\left(\mathcal{N}+\frac{2}{\pi \sqrt{3}} \mathcal{N}^{2}\right)
\end{aligned}
$$

- By the analog of lemma 4 for $\mathbb{D P}$-model for every $x_{k} \in \operatorname{Int}_{C_{\mathrm{m}}}$ and $x_{l} \in \operatorname{Int}_{C_{\mathrm{m}}}$ we have

$$
\left(t_{k}^{0}-t_{l}^{0}\right)^{2} \leq \mathcal{N} \sum_{\Pi_{i j} \in C_{\mathrm{m}}}\left(t_{i}^{0}-t_{j}^{0}\right)^{2}
$$

- Using the estimates (4.15), (4.16) and (A.4) in proposition 4.2 we have

$$
\begin{aligned}
\widehat{a} & \leq \frac{1}{4 L} \sum_{\Pi_{i j}}\left[g_{i j}^{0}+\frac{|\ln (1-\beta)|+\pi+\ln 2}{6}\right]\left(t_{i}^{0}-t_{j}^{0}\right)^{2} \\
& +\frac{1}{4 L} \frac{2}{\sqrt{1-\beta^{2}}} \sum_{\Delta_{i j k}, \beta_{j k}^{\max }=\max \left(\beta_{i j}^{\max }, \beta_{k j}^{\max }, \beta_{k i}^{\max }\right)}\left(\left(t_{i}-t_{j}\right)^{2}+\left(t_{k}-t_{i}\right)^{2}\right) \\
& \leq \frac{1}{4 L} \sum_{\Pi_{i j}, e_{i j} \notin \mathbb{D}_{\delta}} g_{i j}^{0}\left(t_{i}^{0}-t_{j}^{0}\right)^{2}+\frac{1}{4 L} \sum_{\Pi_{i j}, e_{i j} \in \mathbb{D}_{\delta}}\left(t_{i}^{0}-t_{j}^{0}\right)^{2} \\
& \times\left[g_{i j}^{0}+\mathcal{N}\left(\mathcal{N}+\frac{2}{\pi \sqrt{3}} \mathcal{N}^{2}\right)\left(22 \frac{2}{\sqrt{1-\beta^{2}}}+3 \frac{|\ln (1-\beta)|+\pi+\ln 2}{6}\right)\right] \\
& \leq \frac{1}{4 L} \sum_{\Pi_{i j}, e_{i j} \notin \mathbb{D}_{\delta}} g_{i j}^{0}\left(t_{i}^{0}-t_{j}^{0}\right)^{2}+\frac{1}{4 L} \sum_{\Pi_{i j}, e_{i j} \in \mathbb{D}_{\delta}} g_{i j}^{0}\left(1+2.56 \mathcal{N}^{4} \sqrt{\frac{\delta}{R}}\right)\left(t_{i}^{0}-t_{j}^{0}\right)^{2} \\
& \leq\left(1+2.56 \mathcal{N}^{4} \sqrt{\frac{\delta}{R}}\right) \frac{1}{4 L} \sum_{\Pi_{i j}} g_{i j}^{0}\left(t_{i}^{0}-t_{j}^{0}\right)^{2}=\left(1+2.56 \mathcal{N}^{4} \sqrt{\frac{\delta}{R}}\right) I_{0} .
\end{aligned}
$$

Here $2.56 \mathcal{N}^{4}$ is a uniform upper bound on

$$
\sqrt{\frac{R}{\delta}} \frac{1}{g_{i j}^{0}}\left(\mathcal{N}+\frac{2}{\pi \sqrt{3}} \mathcal{N}^{2}\right)\left(22 \frac{2}{\sqrt{1-\beta^{2}}}+3 \frac{|\ln (1-\beta)|+\pi+\ln 2}{6}\right) .
$$

Therefore

$$
I_{0} \leq \widehat{a} \leq\left(1+2.56 \mathcal{N}^{4} \sqrt{\frac{\delta}{R}}\right) I_{0}
$$


or

$$
0 \leq \widehat{a}-I_{0} \leq 2.56 \mathcal{N}^{4} \sqrt{\frac{\delta}{R}} I_{0}
$$

Hence

$$
\frac{\left|\widehat{a}-I_{0}\right|}{I_{0}} \leq 2.56 \mathcal{N}^{4} \sqrt{\frac{\delta}{R}}
$$

Theorem 4.4 Under the $\delta$ - $\mathcal{N}$ close packing condition for $\delta \leq R / 32$ the relative error

$$
\frac{|\widehat{a}-I|}{I} \leq 9.82 \mathcal{N}^{4} \sqrt{\frac{\delta}{R}}
$$

where the effective conductivity

$$
\begin{aligned}
& \widehat{a}=\frac{1}{4 L} \int_{Q_{p}}|\nabla \phi|^{2} d x, \\
& \frac{1}{4 L} \int_{Q_{p}}|\nabla \phi|^{2} d x=\frac{1}{4 L} \min _{\tilde{\phi} \in V_{p}} \int_{Q_{p}}|\nabla \tilde{\phi}|^{2} d x
\end{aligned}
$$

and the energy of the $\mathbb{D}$ - model

$$
\begin{aligned}
& I \equiv I(\mathbf{t})=\frac{1}{4 L} \sum_{\Pi_{i j}} g_{i j}\left(t_{i}-t_{j}\right)^{2} \\
& \frac{1}{4 L} \sum_{\Pi_{i j}} g_{i j}\left(t_{i}-t_{j}\right)^{2}=\frac{1}{4 L} \min _{\tilde{\mathbf{t}}=\left\{\tilde{t}_{1}, \tilde{t}_{2}, \ldots \tilde{t}_{M}\right\}} \sum_{\Pi_{i j}} g_{i j}\left(\tilde{t}_{i}-\tilde{t}_{j}\right)^{2} .
\end{aligned}
$$

Proof: Using (4.9) and (A.17) we have

$$
\frac{1}{4 L} \sum_{\Pi_{i j}} g_{i j}^{0}\left(t_{i}^{0}-t_{j}^{0}\right)^{2} \equiv I_{0}\left(\mathbf{t}^{0}\right) \leq I(\mathbf{t})
$$

and

$$
I(\mathbf{t}) \leq \frac{1}{4 L} \sum_{\Pi_{i j}} g_{i j}^{0}\left(t_{i}^{0}-t_{j}^{0}\right)^{2}+\frac{1}{4 L} \sum_{\Pi_{i j}}\left|g_{i j}-g_{i j}^{0}\right|\left(t_{i}^{0}-t_{j}^{0}\right)^{2}
$$

The length of the largest neck $\delta_{i j}$ can be bounded by means of the maximal size of the minimal cycles of the $\delta$-subgraph $\mathbb{D}_{\delta}$. We have

$$
\delta_{i j} \leq \mathcal{N}(R+\delta / 2) \leq \frac{5}{4} \mathcal{N} R
$$

Similar to the proof of (4.11), by (3.31), (3.18), (4.21) and (A.17) inequality (4.20) 
gives

$$
\begin{aligned}
& I(\mathbf{t}) \leq \frac{1}{4 L} \sum_{\Pi_{i j}, e_{i j} \notin \mathbb{D}_{\delta}} g_{i j}^{0}\left(t_{i}^{0}-t_{j}^{0}\right)^{2}+\frac{1}{4 L} \sum_{\Pi_{i j}, e_{i j} \in \mathbb{D}_{\delta}}\left(t_{i}^{0}-t_{j}^{0}\right)^{2} \\
& \times\left[g_{i j}^{0}+3 \mathcal{N}\left(\mathcal{N}+\frac{2}{\pi \sqrt{3}} \mathcal{N}^{2}\right)\left(\pi+\pi \frac{3}{2 \sqrt{2}} \sqrt{\frac{5}{4}} \mathcal{N}+6\right)\right] \\
& \leq \frac{1}{4 L} \sum_{\Pi_{i j}, e_{i j} \notin \mathbb{D}_{\delta}} g_{i j}^{0}\left(t_{i}^{0}-t_{j}^{0}\right)^{2}+\frac{1}{4 L} \sum_{\Pi_{i j}, e_{i j} \in \mathbb{D}_{\delta}} g_{i j}^{0}\left(1+9.82 \mathcal{N}^{4} \sqrt{\frac{\delta}{R}}\right)\left(t_{i}^{0}-t_{j}^{0}\right)^{2} \\
& \leq\left(1+9.82 \mathcal{N}^{4} \sqrt{\frac{\delta}{R}}\right) \frac{1}{4 L} \sum_{\Pi_{i j}} g_{i j}^{0}\left(t_{i}^{0}-t_{j}^{0}\right)^{2}=\left(1+9.82 \mathcal{N}^{4} \sqrt{\frac{\delta}{R}}\right) I_{0}\left(\mathbf{t}^{\mathbf{0}}\right) .
\end{aligned}
$$

Therefore

$$
I(\mathbf{t}) \leq\left(1+9.82 \mathcal{N}^{4} \sqrt{\frac{\delta}{R}}\right) I_{0}\left(\mathbf{t}^{\mathbf{0}}\right) .
$$

By (4.20), (4.22) and (4.17)

$$
\begin{aligned}
& |\widehat{a}-I(\mathbf{t})| \leq \max \left(9.82 \mathcal{N}^{4}, 2.56 \mathcal{N}^{4}\right) \sqrt{\frac{\delta}{R}} I_{0}\left(\mathbf{t}^{\mathbf{0}}\right) \\
& \leq 9.82 \mathcal{N}^{4} \sqrt{\frac{\delta}{R}} I_{0}\left(\mathbf{t}^{\mathbf{0}}\right) \leq 6 \mathcal{N}^{4} \sqrt{\frac{\delta}{R}} I(\mathbf{t}) .
\end{aligned}
$$

Hence

$$
\frac{|\widehat{a}-I|}{I} \leq 9.82 \mathcal{N}^{4} \sqrt{\frac{\delta}{R}}
$$

The constants $2.56 \mathcal{N}^{4} \sqrt{\delta / R}$ in (4.11) and $9.82 \mathcal{N}^{4} \sqrt{\delta / R}$ in (4.18) are very rough upper bounds on the relative errors and they could be significantly improved by more careful analysis. We give now a better estimate on the relative errors in two special cases: $\mathcal{N}=3$ and $\mathcal{N}=4$.

Theorem 4.5 If $\mathcal{N}=3$ and $\delta \leq R / 32$

$$
\frac{\left|\widehat{a}-I_{0}\right|}{I_{0}} \leq 3.84 \sqrt{\frac{\delta}{R}}
$$

where the effective conductivity

$$
\begin{aligned}
& \widehat{a}=\frac{1}{4 L} \int_{Q_{p}}|\nabla \phi|^{2} d x \\
& \frac{1}{4 L} \int_{Q_{p}}|\nabla \phi|^{2} d x=\frac{1}{4 L} \min _{\tilde{\phi} \in V_{p}} \int_{Q_{p}}|\nabla \tilde{\phi}|^{2} d x
\end{aligned}
$$


and the energy of the $\mathbb{D P}$-model

$$
\begin{aligned}
& I_{0} \equiv I_{0}(\mathbf{t})=\frac{1}{4 L} \sum_{\Pi_{i j}} g_{i j}^{0}\left(t_{i}^{0}-t_{j}^{0}\right)^{2}, \\
& \frac{1}{4 L} \sum_{\Pi_{i j}} g_{i j}^{0}\left(t_{i}^{0}-t_{j}^{0}\right)^{2}=\frac{1}{4 L} \min _{\tilde{\mathbf{t}}^{0}=\left\{\tilde{t}_{1}^{0}, \tilde{t}_{2}^{0}, \ldots \tilde{t}_{M}^{0}\right\}} \sum_{\Pi_{i j}} g_{i j}^{0}\left(\tilde{t}_{i}^{0}-\tilde{t}_{j}^{0}\right)^{2} .
\end{aligned}
$$

Proof: Using (A.22) and (A.2) in the variational bound (4.7) (proposition 4.2) we have

$$
\begin{aligned}
\widehat{a} & \leq \frac{1}{4 L} \sum_{\Pi_{i j}}\left[g_{i j}^{0}+\frac{|\ln (1-\beta)|+\pi+\ln 2}{6}+2 \frac{4}{\sqrt{3}}\left(1+\frac{\delta}{2 R}\right)\right]\left(t_{i}^{0}-t_{j}^{0}\right)^{2} \\
& \leq\left(1+3.84 \sqrt{\frac{\delta}{R}}\right) \frac{1}{4 L} \sum_{\Pi_{i j}} g_{i j}^{0}\left(t_{i}^{0}-t_{j}^{0}\right)^{2}=\left(1+3.84 \sqrt{\frac{\delta}{R}}\right) I_{0}\left(\mathbf{t}^{\mathbf{0}}\right) .
\end{aligned}
$$

Hence

$$
\left|\widehat{a}-I_{0}\left(\mathbf{t}^{\mathbf{0}}\right)\right| \leq 4 \sqrt{\frac{\delta}{R}} I_{0}\left(t^{0}\right), \text { or } \frac{\left|\widehat{a}-I_{0}\right|}{I_{0}} \leq 4 \sqrt{\frac{\delta}{R}}
$$

Theorem 4.6 If $\mathcal{N}=3$ and $\delta \leq R / 32$

$$
\frac{|\widehat{a}-I|}{I} \leq 8.68 \sqrt{\frac{\delta}{R}}
$$

where the effective conductivity

$$
\begin{aligned}
& \widehat{a}=\frac{1}{4 L} \int_{Q_{p}}|\nabla \phi|^{2} d x \\
& \frac{1}{4 L} \int_{Q_{p}}|\nabla \phi|^{2} d x=\frac{1}{4 L} \min _{\tilde{\phi} \in V_{p}} \int_{Q_{p}}|\nabla \tilde{\phi}|^{2} d x
\end{aligned}
$$

and the energy of the $\mathbb{D}$-model

$$
\begin{aligned}
& I \equiv I(\mathbf{t})=\frac{1}{4 L} \sum_{\Pi_{i j}} g_{i j}\left(t_{i}-t_{j}\right)^{2}, \\
& \frac{1}{4 L} \sum_{\Pi_{i j}} g_{i j}\left(t_{i}-t_{j}\right)^{2}=\frac{1}{4 L} \min _{\tilde{\mathbf{t}}=\left\{\tilde{t}_{1}, \tilde{t}_{2}, \ldots . \tilde{t}_{M}\right\}} \sum_{\Pi_{i j}} g_{i j}\left(\tilde{t}_{i}-\tilde{t}_{j}\right)^{2} .
\end{aligned}
$$

Proof: By (A.17) and (4.9)

$$
I_{0}\left(\mathbf{t}^{\mathbf{0}}\right) \leq I(\mathbf{t}) \leq \frac{1}{4 L} \sum_{\Pi_{i j}}\left[g_{i j}^{0}+8.68 \frac{\delta}{R}\right]\left(t_{i}^{0}-t_{j}^{0}\right)^{2}=\left(1+3.84 \sqrt{\frac{\delta}{R}}\right) I_{0}\left(\mathbf{t}^{\mathbf{0}}\right) .
$$


Therefore

$$
|\widehat{a}-I(\mathbf{t})| \leq \max (8.68,3.84) \sqrt{\frac{\delta}{R}} I_{0}\left(\mathbf{t}^{\mathbf{0}}\right) \leq 8.68 \sqrt{\frac{\delta}{R}} I(\mathbf{t}) .
$$

Theorem 4.7 If $\mathcal{N}=4$ and $\delta \leq R / 32$

$$
\frac{\left|\widehat{a}-I_{0}\right|}{I_{0}} \leq 12.73 \sqrt{\frac{\delta}{R}}
$$

where the effective conductivity

$$
\begin{aligned}
& \widehat{a}=\frac{1}{4 L} \int_{Q_{p}}|\nabla \phi|^{2} d x \\
& \frac{1}{4 L} \int_{Q_{p}}|\nabla \phi|^{2} d x=\frac{1}{4 L} \min _{\tilde{\phi} \in V_{p}} \int_{Q_{p}}|\nabla \tilde{\phi}|^{2} d x
\end{aligned}
$$

and the energy of the $\mathbb{D P}$-model

$$
\begin{aligned}
& I_{0} \equiv I_{0}(\mathbf{t})=\frac{1}{4 L} \sum_{\Pi_{i j}} g_{i j}^{0}\left(t_{i}^{0}-t_{j}^{0}\right)^{2}, \\
& \frac{1}{4 L} \sum_{\Pi_{i j}} g_{i j}^{0}\left(t_{i}^{0}-t_{j}^{0}\right)^{2}=\frac{1}{4 L} \min _{\tilde{\mathbf{t}}^{0}=\left\{\tilde{t}_{1}^{0}, \tilde{t}_{2}^{0}, \ldots \tilde{t}_{M}^{0}\right\}} \sum_{\Pi_{i j}} g_{i j}^{0}\left(\tilde{t}_{i}^{0}-\tilde{t}_{j}^{0}\right)^{2} .
\end{aligned}
$$

Proof: Using (A.23) and (A.3) in the variational bound (4.7) (proposition 4.2) we have

$$
\begin{aligned}
\widehat{a} & \leq \frac{1}{4 L} \sum_{\Pi_{i j}}\left[g_{i j}^{0}+\frac{|\ln (1-\beta)|+\pi+\ln 2}{6}+6 \sqrt{2}\left(1+\frac{\delta}{2 R}\right)\right]\left(t_{i}^{0}-t_{j}^{0}\right)^{2} \\
& \leq\left(1+12.73 \sqrt{\frac{\delta}{R}}\right) \frac{1}{4 L} \sum_{\Pi_{i j}} g_{i j}^{0}\left(t_{i}^{0}-t_{j}^{0}\right)^{2}=\left(1+12.73 \sqrt{\frac{\delta}{R}}\right) I_{0}\left(\mathbf{t}^{0}\right) .
\end{aligned}
$$

Hence

$$
\left|\widehat{a}-I_{0}\left(\mathbf{t}^{\mathbf{0}}\right)\right| \leq 12.73 \sqrt{\frac{\delta}{R}} I_{0}\left(\mathbf{t}^{\mathbf{0}}\right), \text { or } \frac{\left|\widehat{a}-I_{0}\right|}{I_{0}} \leq 12.73 \sqrt{\frac{\delta}{R}} .
$$

Theorem 4.8 If $\mathcal{N}=4$ and $\delta \leq R / 32$

$$
\frac{|\widehat{a}-I|}{I} \leq 32.74 \sqrt{\frac{\delta}{R}}
$$

where the effective conductivity

$$
\begin{aligned}
& \widehat{a}=\frac{1}{4 L} \int_{Q_{p}}|\nabla \phi|^{2} d x \\
& \frac{1}{4 L} \int_{Q_{p}}|\nabla \phi|^{2} d x=\frac{1}{4 L} \min _{\tilde{\phi} \in V_{p}} \int_{Q_{p}}|\nabla \tilde{\phi}|^{2} d x
\end{aligned}
$$


and the energy of the $\mathbb{D}$-model

$$
\begin{aligned}
& I \equiv I(\mathbf{t})=\frac{1}{4 L} \sum_{\Pi_{i j}} g_{i j}\left(t_{i}-t_{j}\right)^{2} \\
& \frac{1}{4 L} \sum_{\Pi_{i j}} g_{i j}\left(t_{i}-t_{j}\right)^{2}=\frac{1}{4 L} \min _{\tilde{\mathbf{t}}=\left\{\tilde{t}_{1}, \tilde{t}_{2}, \ldots \tilde{t}_{M}\right\}} \sum_{\Pi_{i j}} g_{i j}\left(\tilde{t}_{i}-\tilde{t}_{j}\right)^{2} .
\end{aligned}
$$

Proof: When $\mathcal{N}=4$ the maximal $\delta_{i j}$ is $\sqrt{2}(2 R+\delta)-2 R$. By (A.17) and (4.9)

$$
I_{0}\left(\mathbf{t}^{\mathbf{0}}\right) \leq I(\mathbf{t}) \leq \frac{1}{4 L} \sum_{\Pi_{i j}}\left[g_{i j}^{0}+32.74 \frac{\delta}{R}\right]\left(t_{i}^{0}-t_{j}^{0}\right)^{2}=\left(1+4.3 \sqrt{\frac{\delta}{R}}\right) I_{0}\left(\mathbf{t}^{\mathbf{0}}\right) .
$$

Therefore

$$
|\widehat{a}-I(\mathbf{t})| \leq \max (32.74,12.73) \sqrt{\frac{\delta}{R}} I_{0}\left(\mathbf{t}^{\mathbf{0}}\right) \leq 32.74 \sqrt{\frac{\delta}{R}} I(\mathbf{t}) .
$$

Remark 5 Asymptotically as $\delta \rightarrow 0$ both models $(\mathbb{D}$-model and $\mathbb{D P}$-model) give the same values of the effective conductivity, in the sense that

$$
\begin{gathered}
I(\mathbf{t})=O\left(\sqrt{\frac{R}{\delta}}\right),|I(\mathbf{t})-\widehat{a}|=O(1), \\
I_{0}\left(\mathbf{t}^{\mathbf{0}}\right)=O\left(\sqrt{\frac{R}{\delta}}\right),\left|I\left(\mathbf{t}^{\mathbf{0}}\right)-\widehat{a}\right|=O(1), \\
\left.\mid I_{(} \mathbf{t}^{\mathbf{0}}\right)-I(\mathbf{t}) \mid=O(1), \text { as } \delta \rightarrow 0 .
\end{gathered}
$$

Since in the $\mathbb{D P}$-model we take into account more geometry of the original continuous problem, it may seem that the $\mathbb{D P}$-model gives a better approximation to the effective conductivity than the $\mathbb{D}$-model. However, due to the errors that arise when we integrate perpendicular to the necks (see (A.7)) the errors of both models are compatible. More specifically, the $\mathbb{D}$-model approximates the Dirichlet's integral in a neck

$$
\int_{\Pi_{i j}}|\nabla \phi|^{2} d x=\int_{\Pi_{i j}}\left(\frac{\partial \phi}{\partial x}\right)^{2} d x+\int_{\Pi_{i j}}\left(\frac{\partial \phi}{\partial y}\right)^{2} d x
$$

by $g_{i j}\left(t_{i}-t_{j}\right)^{2}$, where $g_{i j}$ is given by the Keller's asymptotic formula (3.5). This asymptotic formula neglects the value of the first term on the right-hand side of (4.28) and approximates the second term of (4.28). On the other hand, in the $\mathbb{D P}$-model we compute the second term of the Dirichlet's integral (4.28) exactly, but we neglect the first term of it. When $\delta \rightarrow 0$ the value of this second term in (4.28) is compatible to the error of the Keller's approximation (3.5) of the first term, hence the $\mathbb{D P}$-model does not provide with the approximation of the effective conductivity which is better than the one of the $\mathbb{D}$-model. This means that for the purpose of estimating the effective conductivity of a composite where inclusions are almost touching each other both models are equivalently good; however, the $\mathbb{D P}$-model also provides with a rigorous lower variational bound on the effective conductivity even if $\delta$ is not small, because $I_{0}\left(\mathbf{t}^{\mathbf{0}}\right) \leq \widehat{a}$. 


\subsection{A numerical illustration}

The main goal of this paper is to give a rigorous quantitative justification of discrete network model (1.5) by means of a priori error estimates. However, the use of our trial functions for the upper and the lower bounds gives a numerical a posteriori error. We must solve the discrete network problem (3.25), construct the trial functions $\phi \in V_{p}$ (see section 4.1) and $\mathbf{v} \in W_{p}$ (see section 4.2) and evaluate explicitly the left-hand side and the right-hand side of the upper and lower bound (2.16). The evaluation of this dual bound is not computationally expensive, because we use simple trial functions - they are given by explicit analytic formulas on the necks and they are linear interpolations on the triangles. This section implements this idea for numerical simulations of a randomized hexagonal lattice.

The algorithm for these simulations consists of three parts:

- An algorithm for a numerical simulation of a randomized hexagonal distribution of disks.

- An algorithm for a numerical evaluation of the dual variational bounds.

- Statistics.

Let us discuss these three parts in more detail.

Distribution of disks. The distribution of disks is implemented by randomization of a periodic hexagonal lattice of disks of equal radii $R_{i}=R=0.02$ on a square domain $[-1,1] \times[-1,1]$, and then removal of some fraction of these disks from this distribution. More specifically:

- Choose a volume fraction $f$.

- By formula (3.12) determine the corresponding interparticle distance $\delta$ for the chosen volume fraction $f$.

- Distribute disks of radius $R_{i}=R=0.02$ with the computed interparticle distance $\delta$ periodically on a square domain $[-1,1] \times[-1,1]$ as in figure 3.2. The centers of the boundary disks may not lie exactly on the boundary, but as we have already remarked this makes insignificant corrections to the effective conductivity.

- Randomize the hexagonal periodic distribution of disks by shifting the centers of all the disks by a random vector. We chose to shift each centers of a disk independently by a vector with a length that has a uniform distribution on an interval $[0,1 / 3 \delta]$ and with a direction that has a uniform distribution on $[0,2 \pi]$. This randomization guarantees that the distance between neighbors $\delta_{i j} \in[1 / 3 \delta, 5 / 3 \delta]$.

- Choose a volume fraction $f_{r} f_{r}<f$ of disks to be removed. Compute the number of disks to be removed and then remove this number randomly with uniform probability. This removal procedure typically creates holes of size $\mathcal{N}=$ 6 , if $f_{r}$ is small. 


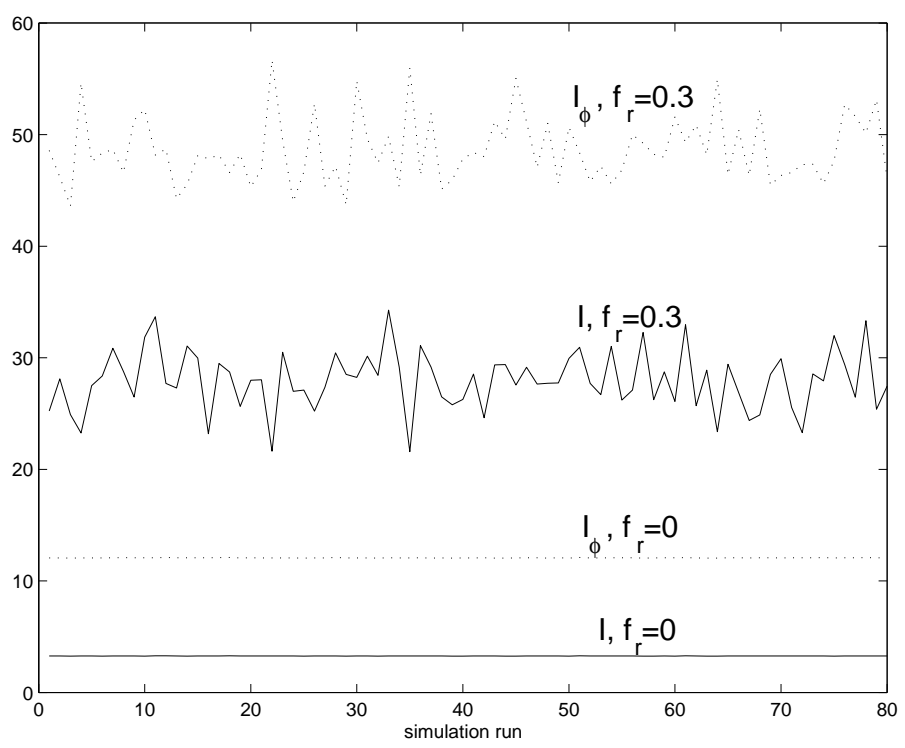

Figure 4.3: $I_{0}$ (solid lines) and $I_{\phi}$ (dotted lines) for $f_{0}=.605$ for simulation runs $1, \ldots, 80$. Lower two lines for $f_{r}=0$ - no holes. Upper two lines for $f_{r}=.3$ - large holes.

For fixed $f$ and $f_{r}$ this algorithm creates a distribution of disks with the volume fraction $f_{0}=f-f_{r}$ and with a characteristic interparticle distance $\delta$ computed by (3.12).

Computation of bounds. For a given distribution of disks we compute $I_{0}=I_{0}\left(\mathbf{t}^{\mathbf{0}}\right)$ (formula (3.35)), the energy of the $\mathbb{D P}$-model. After the energy $I_{0}$ of the $\mathbb{D P}$-model is computed, we also compute the trial function $\phi$ for the upper bound as in section 4.2 , and then we compute

$$
I_{\phi}=\frac{1}{4 L} \int_{Q_{p}}|\nabla \phi|^{2} d \mathbf{x}
$$

for this trial function. Therefore we have

$$
\begin{aligned}
& I_{0} \leq \widehat{a} \text { by construction in section } 4.1 \\
& \widehat{a} \leq I_{\phi} \text { by construction in section } 4.2 \text {. }
\end{aligned}
$$

Hence $I_{0}$ and $I_{\phi}$ are a posteriori lower and upper bounds respectively for the effective conductivity of a composite with a given distribution of disks. The details of these numerical computations are as follows:

- We used MATLAB for all our numerical computations.

- The fluxes $g_{i j}^{0}$ are computed by (3.4) using explicit formulas (A.12) and (A.18).

- The values of the discrete potentials $\mathbf{t}^{\mathbf{0}}$ (solution of 3.34) for the discrete energies $I$ and $I_{0}$ are computed using $p c g$ - Preconditioned Conjugate Gradient method with the number of iterations 90 and the relative residual of order $10^{-7}$. 
- After $\mathbf{t}^{\mathbf{0}}$ are determined, $I_{\phi}$ is evaluated using explicit formulas (A.8) (A.21).

The computation of $I_{0}$ and $I_{\phi}$ is fast, therefore it allows to study the statistics of the effective conductivity.

Statistics. The simulations are done with the 0.05 increments of $f_{r}$. For fixed $f$ and $f_{r}$ there were 80 simulations. For the mean we use the notation

$$
\mathbb{E}(I)=1 / n \sum_{k=1}^{n} I^{k}
$$

where $I^{k}$ is the result of $k$-th simulation with fixed $f$ and $f_{r}$ and the number of simulations $n=80$.

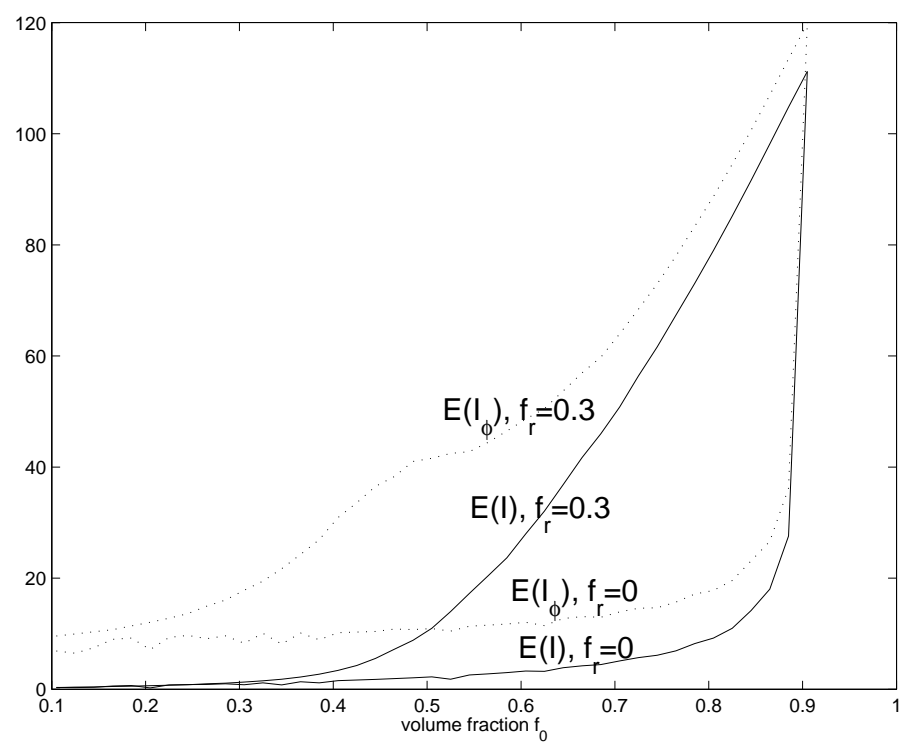

Figure 4.4: $\mathbb{E}\left(I_{0}\right)$ (solid lines) and $\mathbb{E}\left(I_{\phi}\right)$ (dotted lines) as functions of the volume fraction $f_{0}=0.105, \ldots 0.905$. Lower two lines for the case with no holes. Upper two lines for the case with holes.

Results. Here we present the results of the numerical simulations that show the dependence of the effective conductivity on the presence of holes in the matrix.

On figure 4.3 we plot $I_{0}$ (solid lines) and $I_{\phi}$ (dotted lines) for all 80 simulations when the volume fraction of the inclusions is fixed $f_{0}=0.605$ and $f_{r}$ takes two values $f_{r}=0$ and $f_{r}=.3$. The case $f_{r}=0$ corresponds to the case when there are no holes in the material. The case $f_{r}=.3$ corresponds to almost maximal possible number of holes for this volume fraction in the sense that before removal of disks the volume fraction $f=0.905$ is very close to the densest possible (hexagonal) packing. Indeed, the volume fraction of the periodic hexagonal packing when disks touch is $\pi \sqrt{3} / 6=0.9068 \ldots$ Observe that for the same total volume fraction $f_{0}=0.605$ for all numerical simulations a distribution of disks with holes has greater effective 
conductivity than a distribution of disks without holes, because the lower bound $I_{0}$ on the effective conductivity for a distribution of disks with holes is always larger than the upper bound $I_{\phi}$ on the effective conductivity for a distribution of disks without holes.

On figure 4.4 we plot $\mathbb{E}\left(I_{0}\right)$ (solid lines) and $\mathbb{E}\left(I_{\phi}\right)$ (dotted lines) as functions of the volume fraction $f_{0}=0.105, \ldots 0.905$. The volume fraction of removed disks $f_{r}$ takes two values $f_{r}=0$ and $f_{r}=.3$. Observe that in the presence of holes the a posteriori error of the network approximation $I_{\phi}-I_{0}$ is significantly larger than in the case when there are no holes. For example when $f_{0}=.5$ in the presence of holes the a posteriori error is 3.5 times larger than the error in the case when there are no holes.

If the total volume fraction of the inclusions is fixed, then the increase of the volume fraction of holes in the material implies that the interparticle distance $\delta$ decreases. Hence the percolation effects play a more significant role. Therefore we expect that for highly packed composites the relative error of our discrete model is smaller in the case when there are holes compared with the case when there is no holes. Indeed, when $f_{0}>.6$ the relative a posteriori error for composites with holes is up to 8 times better than relative error for composites with no holes for the same volume fraction. When $f_{0} \leq .35$ the effective conductivities of a material with holes and of a material without holes are numerically very close, at least the computed a posteriori error does not allow to distinguish between these composites. For such volume fractions there are no percolation effects in both cases.

Remark 6 Let us compare the a posteriori error estimates discussed in this section and the a priori error estimates given in section 4.3. Suppose we want to compute the maximal interparticle distance $\delta$ such that the error of the discrete network approximation is less than $10 \%$. Consider the case of a quasi-hexagonal lattice $(\boldsymbol{\mathcal { N }}=3)$. From (4.23) the a priori error estimate is

$$
\sqrt{\frac{\delta}{R}} \leq .1, \text { therefore } \delta \leq 6.78 \cdot 10^{-4} R .
$$

So the a priori error estimate guarantees a $10 \%$ accuracy if $\delta$ is about 1500 times smaller, than the radius of the disks $R$.

The a posteriori error estimate can be computed from our numerical simulations for a quasi-hexagonal distribution of disks with no holes (see the lines that correspond to $f_{r}=0$ on figure 4.4). These simulations show, that a $10 \%$ accuracy is achieved if $\delta$ is about 250 times smaller, than the radius of the disks $R$.

Therefore the use of the a posteriori error widens the range of the characteristic distance $\delta$, where the discrete network gives a good approximation.

\section{Conclusions}

In this paper we derived an explicit a priori error estimate for the network approximation (the $\mathbb{D}$-model) introduced in [4] for the effective conductivity of a high contrast highly packed composite under the so-called $\delta$-close packing-conditions. Furthermore, the approach developed in this paper allowed us to consider much more generic geometrical arrays of the particles which satisfy the so-called $\delta$ - $\mathcal{N}$ close packing condition. Roughly speaking the first condition means that all the neighboring particles 
are closely spaced whereas the second condition allows for strongly non-uniform geometrical arrays when a significant fraction of the particles does not participate in the conducting cluster. We have shown quantitatively how such nonuniformity affects the error estimate and therefore the quality of the approximation.

We have also introduced and justified the modified network approximation (the $\mathbb{D P}$ model) which generalizes the network approximation from [4]. This modified network approximation is no longer asymptotic in nature. Roughly speaking we decompose the Dirichlet integral (1.4) for the effective conductivity into two parts: the network approximation, which is a quadratic form and the error term. Our modified network approximation accounts for all fluxes between the neighboring particles, where neighbors are defined via Voronoi tessellation. If the fluxes are small (neighbors are not closely spaced), then the corresponding coefficients in the modified network approximation (1.9) are not significant but unlike [4] we do not need to introduce any cut-off distance in the numerical implementation. For the both network approximations (the $\mathbb{D}$-model and the $\mathbb{D P}$-model) the error term is explicitly estimated based on the construction of the trial functions using the triangle-neck partition of the domain. The comparison of the error terms in the two models shows that the a priori estimate in the $\mathbb{D P}$-model is about $2.5-3$ times better than that for the $\mathbb{D}$-model which makes a big difference in practical computations.

Finally we provide model examples of numerical implementation of the network approximation. We observe that irregularity in the geometrical distribution of the inclusions in all simulations consistently lead to a significant (up to 10 times) increase in the effective conductivity at the same total volume fraction of the inclusions. We also observe numerically that our network approximation works better for irregular geometric patterns, which are not quasi-hexagonal, that is when a typical number of nearest neighbors can vary significantly. In our model example for the same volume fraction of inclusions (larger than .6) the the relative a posteriori error estimate in the presence of holes in the conducting cluster is up to 8 times better than for uniformly highly packed composites.

Thus we conclude that both approximations provide a very efficient computational tool for evaluation of the effective properties of high contrast composites, which is capable of capturing the effects of irregular geometrical arrays with a good control of the approximation error. We expect that the method developed in this work will be generalized for more complicated problems of highly packed elastic and fluid composites.

\section{A Appendix. Auxiliary estimates}

\section{A.1 Estimates on the relative neck widths}

Here we give upper and lower bounds on the relative half-neck widths $\beta_{i j}^{\max }$ and $\beta_{i j}^{\min }$. Recall that ( see (3.17))

$$
\beta_{i j}^{\max }=\max \left(\left|S_{1}\right| / R,\left|S_{2}\right| / R\right), \beta_{i j}^{\min }=\min \left(\left|S_{1}\right| / R,\left|S_{2}\right| / R\right) .
$$

Lemma 5

$$
\beta_{i j}^{\min } \geq \max \left(0,1-\frac{1}{2}\left(1+\frac{\delta_{i j}}{2 R}\right)^{2}\right)
$$




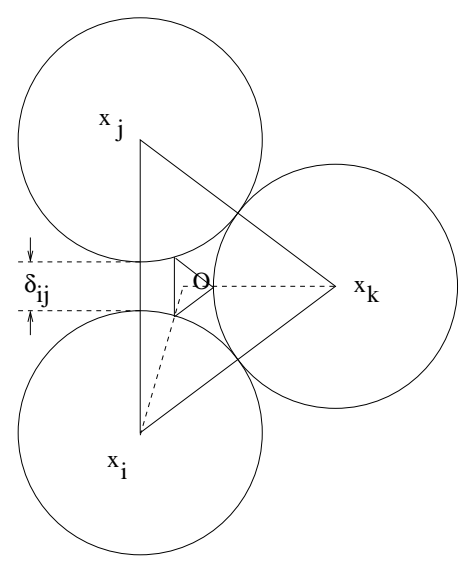

Figure A.1: The worst case scenario for the lower bound on $\beta_{i j}^{\min }$.

Proof: For any distribution of centers $x_{i}, x_{j}$ and $x_{k} \beta_{i j}^{\text {min }}$ is larger than the one shown on figure A.1. In the latter case

$$
\begin{aligned}
& \beta_{i j}^{\min }=\sin \left(\angle O x_{i} x_{j}\right)=\sin \left(\frac{\pi}{2}-2 \angle O x_{k} x_{i}\right) \\
& =1-2 \sin ^{2}\left(\angle O x_{k} x_{i}\right)=1-\frac{1}{2}\left(1+\frac{\delta_{i j}}{2 R}\right)^{2} .
\end{aligned}
$$

Lemma 6 If $\mathbb{N}=3$

$$
\beta_{i j}^{\max } \leq 1-\frac{1}{2}\left(\frac{R}{R+\delta / 2}\right)^{2}
$$

If $\mathbb{N}=4$

$$
\beta_{i j}^{\max } \leq \sqrt{1-\frac{1}{2}\left(\frac{R}{R+\delta / 2}\right)^{2}}
$$

If $\mathbb{N}$ is arbitrary

$$
\beta_{i j}^{\max } \leq 1-2\left(\frac{R}{(R+\delta / 2) \mathcal{N}}\right)^{2}
$$




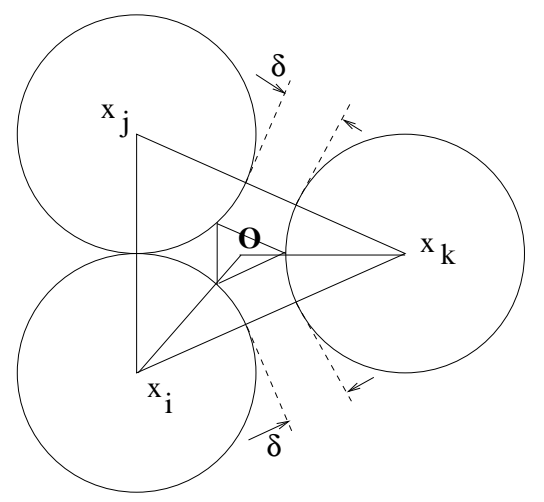

Figure A.2: The worst case scenario for the upper bound on $\beta_{i j}^{\max }$ when $\mathbb{N}=3$.

Proof: The upper bound on $\beta_{i j}^{\max }$ depends on the distribution of other disks. By definition all the vertices $O_{k}$ of the Voronoi tessellation (see figure 3.5) are centers of triangle's $\Delta x_{i} x_{j} x_{k}$ circumcircles (in degenerate cases instead of a triangle $\Delta x_{i} x_{j} x_{k}$ we may have $n$-gons) such that no other vertices $x_{i}$ of the Delaunay graph $\mathbb{D}$ are contained inside these circumcircles. The upper bound on $\beta_{i j}^{\max }$ is determined by the estimate on the maximal possible diameter of these circumcircles.

In the case $\mathbb{N}=3$ the largest $\beta_{i j}^{\max }$ is shown on figure A.2. Here the half-neck between the disks $D_{i}$ and $D_{j}$ is the largest possible. Since $\pi / 2-\angle O x_{i} x_{j}=\angle x_{i} x_{k} x_{j}$ therefore

$$
\beta_{i j}=\sin \angle O x_{i} x_{j}=1-2 \sin ^{2} \angle x_{i} x_{k} O=1-2\left(\frac{R}{2 R+\delta}\right)^{2}=1-\frac{1}{2}\left(\frac{R}{R+\delta / 2}\right)^{2}
$$

In the case $\mathbb{N}=4$ the largest half-neck $\beta_{i j}$ between disks $D_{i}$ and $D_{j}$ is shown on figure A.3. In this case $\left|x_{k} x_{i}\right|=\left|x_{l} x_{k}\right|=2 R+\delta$. Therefore the maximal possible diameter of the circumcircle is $\sqrt{2}(2 R+\delta)$. Since $\left|x_{i} x_{j}\right| 2 R$ then

$$
\beta_{i j}^{\max }=\sqrt{1-\left(\frac{R}{\sqrt{2}(2 R+\delta) / 2}\right)^{2}}=\sqrt{1-\frac{1}{2}\left(\frac{R}{R+\delta / 2}\right)^{2}} .
$$




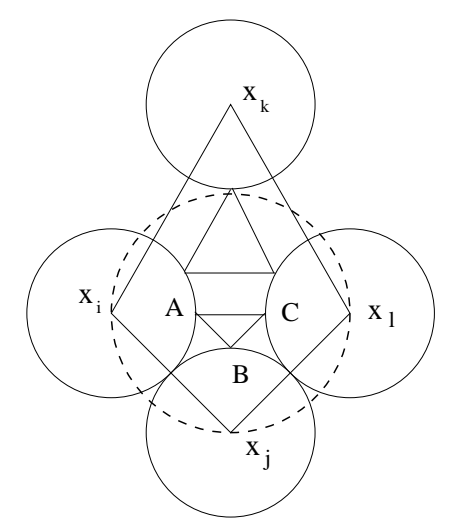

Figure A.3: The worst case scenario for the upper bound on $\beta_{i j}^{\max }$ when $\mathbb{N}=4$.

In the case when $\mathbb{N}$ is arbitrary the diameter of the maximal circumcircle is bounded by a half of the length of the largest minimal cycle. On figure A.4 $x_{i} x_{k}$ is this diameter and we have

$$
\left|x_{i} x_{k}\right| \leq(R+\delta / 2) \mathcal{N} \text {. }
$$

The largest half-neck on figure A.4 is between $x_{i}$ and $x_{j}$. We have

$$
\beta_{i j}^{\max }=\sin \left(\frac{\pi}{2}-\angle x_{i} x_{k} x_{j}\right)=\cos \left(\angle x_{i} x_{k} x_{j}\right) \leq 1-2\left(\frac{R}{(R+\delta / 2) \mathcal{N}}\right)^{2} .
$$

\section{A.2 Gradient estimates inside the necks}

The gradient of $\phi(x, y)$ on the neck $\Pi_{i j}$ is given by

$$
\begin{aligned}
& \partial \phi / \partial x=-\left(t_{j}^{0}-t_{i}^{0}\right) \frac{y H^{\prime}(x)}{H^{2}(x)} \\
& \partial \phi / \partial y=\frac{t_{j}^{0}-t_{i}^{0}}{H(x)}
\end{aligned}
$$




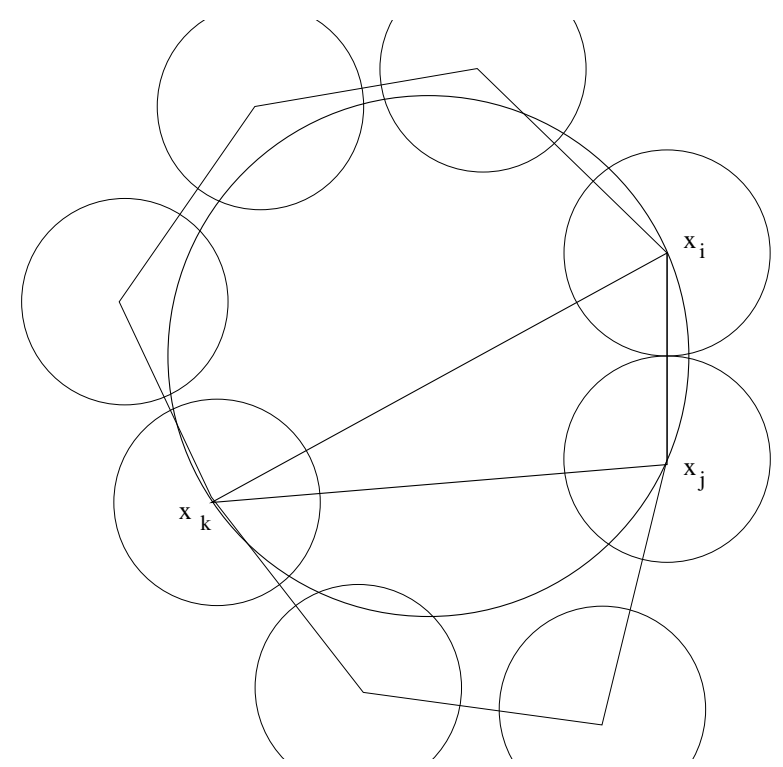

Figure A.4: The worst case scenario for the upper bound on $\beta_{i j}^{\max }$ when $\mathbb{N}$ is arbitrary.

By (A.5)

$$
\int_{\Pi_{i j}}(\partial \phi / \partial y)^{2} d x d y=\int_{S_{1}}^{S_{2}} \frac{\left(t_{j}^{0}-t_{i}^{0}\right)^{2}}{H^{2}(x)} H(x) d x=\left(t_{j}^{0}-t_{i}^{0}\right)^{2} g_{i j}^{0}
$$

where $g_{i j}^{0}$ is defined in (3.4). Also

$$
\begin{aligned}
& \int_{\Pi_{i j}}(\partial \phi / \partial x)^{2} d x d y=\int_{\Pi_{i j}}\left(t_{j}^{0}-t_{i}^{0}\right)^{2}\left[\frac{y H^{\prime}(x)}{H^{2}(x)}\right]^{2} d x d y \\
& =\left.\int_{S_{1}}^{S_{2}}\left(t_{j}^{0}-t_{i}^{0}\right)^{2}\left[\frac{H^{\prime}(x)}{H^{2}(x)}\right]^{2} \frac{y^{3}}{3}\right|_{-H(x) / 2} ^{H(x) / 2} d x=\frac{1}{12}\left(t_{j}^{0}-t_{i}^{0}\right)^{2} \int_{S_{1}}^{S_{2}} \frac{H^{\prime 2}(x)}{H(x)} d x .
\end{aligned}
$$

Since $H(x)=\delta+2 R-2 \sqrt{R^{2}-x^{2}}$, therefore $H^{\prime}(x)=\frac{2 x}{\sqrt{R^{2}-x^{2}}}$ and

$$
\begin{aligned}
& \frac{H^{\prime 2}(x)}{H(x)}=\frac{4 x^{2}}{R^{2}-x^{2}} \frac{1}{\delta+2 R-2 \sqrt{R^{2}-x^{2}}} \leq \\
& \frac{2}{R^{2}-x^{2}} \frac{x^{2}}{R-\sqrt{R^{2}-x^{2}}}=\frac{2}{R^{2}-x^{2}}\left(R+\sqrt{R^{2}-x^{2}}\right)=\frac{2 R}{R^{2}-x^{2}}+\frac{2}{\sqrt{R^{2}-x^{2}}}
\end{aligned}
$$

$$
\int_{S_{1}}^{S_{2}} \frac{2 R}{R^{2}-x^{2}} d x+\int_{S_{1}}^{S_{2}} \frac{2}{\sqrt{R^{2}-x^{2}}} d x=\left.\left(\ln \frac{R+x}{R-x}+2 \arcsin \frac{x}{R}\right)\right|_{S_{1}} ^{S_{2}}
$$


then from (A.7), (A.8) and (A.9) we have

$$
\begin{aligned}
& \int_{\Pi_{i j}}(\partial \phi / \partial x)^{2} d x d y=\frac{1}{12}\left(t_{j}^{0}-t_{i}^{0}\right)^{2} \int_{-S_{1}}^{S_{2}} \frac{H^{\prime 2}(x)}{H(x)} d x \leq \\
& \frac{1}{12}\left(t_{j}^{0}-t_{i}^{0}\right)^{2}\left(2 \ln \frac{2}{1-\beta_{i j}^{\max }}+2 \pi\right)<\frac{\left|\ln \left(1-\beta_{i j}^{\max }\right)\right|+\pi+\ln 2}{6}\left(t_{j}^{0}-t_{i}^{0}\right)^{2}
\end{aligned}
$$

where $\beta_{i j}^{\max }$ is defined by (3.17). Combining (A.10), (A.6) the value $\int_{\Pi_{i j}}|\nabla \phi|^{2} d \mathbf{x}$ is

$$
\int_{\Pi_{i j}}|\nabla \phi|^{2} d \mathbf{x} \leq\left(t_{j}^{0}-t_{i}^{0}\right)^{2}\left[g_{i j}^{0}+\frac{\left|\ln \left(1-\beta_{i j}^{\max }\right)\right|+\pi+\ln 2}{6}\right] .
$$

\section{A.3 Comparison of fluxes for the two discrete networks}

Here we establish a relation between the relative interparticle flux $g_{i j}$ (3.5) and the specific flux $g_{i j}^{s}$ (3.4). Consider the case of a disk and a quasidisk $R_{i}=R, R_{j}=\infty$.

$$
H(x)=\delta_{i j}+R-\sqrt{R^{2}-x^{2}} .
$$

With the change of variables

$$
\begin{aligned}
& x=R \cos \alpha \\
& S_{k}^{*}=\arcsin \left(S_{k} / R\right), \text { for } k=1,2 \\
& -\pi / 2 \leq S_{k}^{*} \leq \pi / 2 .
\end{aligned}
$$

we have

$$
\begin{aligned}
& g_{i j}^{s}=\int_{S_{1}}^{S_{2}} \frac{d x}{\delta_{i j}+R-\sqrt{R^{2}-x^{2}}}=\int_{S_{1}^{*}}^{S_{2}^{*}} \frac{R \cos \alpha d \alpha}{\delta_{i j}+R-R \cos \alpha} \\
& =\int_{S_{1}^{*}}^{S_{2}^{*}}\left[-1+\left(\delta_{i j}+R\right) \frac{1}{\delta_{i j}+R-R \cos \alpha}\right] d \alpha \\
& =\int_{S_{1}^{*}}^{S_{2}^{*}}\left[-1+\left(\delta_{i j}+R\right) \frac{1}{\delta_{i j}+2 R \sin ^{2} \alpha / 2}\right] d \alpha \\
& =\int_{S_{1}^{*}}^{S_{2}^{*}}\left[-1+2\left(\delta_{i j}+R\right) \frac{1 / \delta_{i j}}{\cos ^{2} \alpha / 2+\left(1+2 R / \delta_{i j}\right) \sin ^{2} \alpha / 2}\right] d \alpha \\
& =\left[-\alpha+2 \frac{\delta_{i j}+R}{\left.\delta_{i j}\left(1+2 R / \delta_{i j}\right)^{-1 / 2} \arctan \left(\left(1+2 R / \delta_{i j}\right)^{1 / 2} \tan (\alpha / 2)\right]\right|_{S_{1}^{*}} ^{S_{2}^{*}}}\right. \\
& =\left[-\alpha+\left.2 \frac{\delta_{i j}+R}{\sqrt{\delta_{i j}^{2}+2 R \delta_{i j}}} \arctan \left(\frac{\sqrt{\delta_{i j}^{2}+2 R \delta_{i j} \tan (\alpha / 2)}}{\delta_{i j}}\right]\right|_{S_{1}^{*}} ^{S_{2}^{*}}\right.
\end{aligned}
$$


For each $k=1,2$

$$
\begin{aligned}
& 0<\frac{\pi}{2}-\arctan \left(\sqrt{\frac{2 R+\delta_{i j}}{\delta_{i j}}} \tan \left(\left|S_{k}^{*}\right| / 2\right)\right)=\arctan \left(\sqrt{\frac{\delta_{i j}}{2 R+\delta_{i j}}} \cot \left(\left|S_{k}^{*}\right| / 2\right)\right) \\
& <\min \left(\sqrt{\frac{\delta_{i j}}{2 R}} \cot \left(\left|S_{k}^{*}\right| / 2\right), \frac{\pi}{2}\right) \leq \min \left(\sqrt{\frac{\delta_{i j}}{2 R}} \frac{1+\sqrt{1-\left(\beta_{i j}^{\text {min }}\right)^{2}}}{\beta_{i j}^{\text {min }}}, \frac{\pi}{2}\right) .
\end{aligned}
$$

Using (A.1) we have

$$
\frac{\pi}{2}-\arctan \left(\sqrt{\frac{2 R+\delta_{i j}}{\delta_{i j}}} \tan \left(\left|S_{k}^{*}\right| / 2\right)\right) \leq \min \left(\sqrt{\frac{\delta_{i j}}{2 R}}\left(5.5+\frac{3 \delta_{i j}}{R}\right), \frac{\pi}{2}\right) .
$$

Also

$$
\begin{aligned}
0 & <\frac{\delta_{i j}+R}{\sqrt{\delta_{i j}^{2}+2 R \delta_{i j}}}-\sqrt{\frac{R}{2 \delta_{i j}}} \\
& =\frac{1}{\sqrt{\delta_{i j}}} \frac{\delta_{i j}+R-\sqrt{R \delta_{i j} / 2+R^{2}}}{\sqrt{\delta+2 R}} \\
& =\sqrt{\delta_{i j}} \frac{3 / 2 R+\delta_{i j}}{\delta_{i j}+R+\sqrt{R \delta_{i j} / 2+R^{2}}} / \sqrt{\delta_{i j}+2 R}<\frac{3}{4 \sqrt{2}} \sqrt{\frac{\delta_{i j}}{R}}
\end{aligned}
$$

and we obtain for any $\delta_{i j}$

$$
\begin{aligned}
& g_{i j}-g_{i j}^{s}=\pi \sqrt{\frac{2 R}{\delta_{i j}}}-\left.\left[-\alpha+2 \frac{\delta_{i j}+R}{\sqrt{\delta_{i j}^{2}+2 R \delta_{i j}}} \arctan \left(\frac{\sqrt{\delta_{i j}^{2}+2 R \delta_{i j}} \tan (\alpha / 2)}{\delta_{i j}}\right)\right]\right|_{S_{1}^{*}} ^{S_{2}^{*}} \\
& =\left.\left[\alpha+\left(2 \frac{\delta_{i j}+R}{\sqrt{\delta_{i j}^{2}+2 R \delta_{i j}}}-\sqrt{\frac{2 R}{\delta_{i j}}}\right) \arctan \left(\frac{\sqrt{\delta_{i j}^{2}+2 R \delta_{i j}} \tan (\alpha / 2)}{\delta_{i j}}\right)\right]\right|_{S_{1}^{*}} ^{S_{2}^{*}} \\
& +\left.\sqrt{\frac{2 R}{\delta_{i j}}}\left[\frac{\pi}{2}-\arctan \left(\frac{\sqrt{\delta_{i j}^{2}+2 R \delta_{i j}} \tan (\alpha / 2)}{\delta_{i j}}\right)\right]\right|_{0} ^{S_{1}^{*}} \\
& +\left.\sqrt{\frac{2 R}{\delta_{i j}}}\left[\frac{\pi}{2}-\arctan \left(\frac{\sqrt{\delta_{i j}^{2}+2 R \delta_{i j}} \tan (\alpha / 2)}{\delta_{i j}}\right)\right]\right|_{0} ^{S_{2}^{*}}
\end{aligned}
$$

Combining (A.14), (A.15) and (A.16) we have

$$
0<g_{i j}-g_{i j}^{s} \leq \pi+\pi \frac{3}{2 \sqrt{2}} \sqrt{\frac{\delta_{i j}}{R}}+\sqrt{\frac{2 R}{\delta_{i j}}} \min \left(\sqrt{\frac{\delta_{i j}}{2 R}}\left(5.5+\frac{3 \delta_{i j}}{R}\right), \frac{\pi}{2}\right)
$$


Combining (A.14), (A.15) and (A.16)

$$
0<g_{i j}-g_{i j}^{s} \leq \pi+\pi \frac{3}{2 \sqrt{2}} \sqrt{\frac{\delta_{i j}}{R}}+\min \left(\left(5.5+\frac{3 \delta_{i j}}{R}\right), \frac{\pi}{2} \sqrt{\frac{2 R}{\delta_{i j}}}\right)
$$

The case of two disks with equal radii $R_{i}=R_{j}=R$ follows from (A.17), because the problem of two disks with equal radii becomes equivalent to solving two problems for a disk and quasidisk with the length $\delta_{i j} / 2$ when we draw the middle-point perpendicular to the line which connects centers of the disks(the $\mathrm{x}$-axis on figure 3.1). Analogous to (A.17) we derive estimates for the case $R_{i}=R_{j}=R$ with

$$
H(x)=\delta_{i j}+2 R-2 \sqrt{R^{2}-x^{2}} .
$$

For such $H(x)$ formula (A.12) is

$$
g_{i j}^{0}=\left.\left[-\frac{\alpha}{2}+\frac{\delta_{i j}+2 R}{\sqrt{\delta_{i j}^{2}+4 R \delta_{i j}}} \arctan \left(\frac{\sqrt{\delta_{i j}^{2}+4 R \delta_{i j}} \tan (\alpha / 2)}{\delta_{i j}}\right)\right]\right|_{S_{1}^{*}} ^{S_{2}^{*}} .
$$

However, these new estimates are more tight than (A.17), therefore we must use (A.17) for an estimate independent of radii of the disks. Observe that the estimate (A.17) is valid for any $\delta_{i j}$. The estimate (A.17) implies that the term $g_{i j}$ is the leading term in the asymptotic expansion of $g_{i j}^{s}$ if $\delta_{i j}$ is small and the width $S_{2}-S_{1}$ is nonsmall. Observe that if $\delta_{i j}$ is small it implies that the width of the neck is non-small (estimate (A.1) on $\beta_{i j}^{\min }$ ).

\section{A.4 Gradient estimates inside the triangles}

Lemma 7

$$
\begin{aligned}
& \int_{\Delta_{i j k}}|\nabla \phi|^{2} d x \leq \frac{2}{\sqrt{1-\beta^{2}}}\left(\left(t_{i}-t_{j}\right)^{2}+\left(t_{k}-t_{i}\right)^{2}\right) \\
& \text { where } \beta=\beta_{j k}^{\max }=\max \left(\beta_{i j}^{\max }, \beta_{k j}^{\max }, \beta_{k i}^{\max }\right) .
\end{aligned}
$$

Proof: By construction the Voronoi tessellation induces the triangle neck partition of the domain $\Pi$. Each triangle is (typically) bounded by three (half)necks. On figure 4.2 we show a typical case with three disks centered at $x_{i}, x_{j}$ and $x_{k}$, three half-necks and the $\triangle A B C \equiv \Delta_{i j k}$ bounded by these half-necks. The potential $\phi$ is defined at the vertices of $\triangle A B C$

$$
\begin{aligned}
& \phi(A)=t_{i} \\
& \phi(B)=t_{j} \\
& \phi(C)=t_{k} .
\end{aligned}
$$

Since $\phi$ is a piecewise continuously differentiable define $\phi$ on $\triangle A B C$ by linear interpolation of these three values. Suppose in $\triangle A B C$ the side $|A C|$ is the longest, and the side $|B C|$ is the shortest ( see figure 4.2). Then $0<\angle x_{j} x_{i} x_{k}=\angle B A C \leq \pi / 3$, 
$1 \leq|A C| /|A B| \leq 2, \beta=\beta_{j k}^{\max }$, and the endvertex of the perpendicular $B C$ on the base $A C$ lies between the points $A$ and $C$. Suppose that $\phi(K)=t_{0}$. Then we have

$$
\frac{t_{0}-t_{i}}{t_{k}-t_{i}}=\frac{|A B| \cos (\angle B A C)}{|A C|},
$$

therefore

$$
t_{0}=t_{i}+\left(t_{k}-t_{i}\right) \frac{|A B| \cos (\angle B A C)}{|A C|}
$$

Denoting by $|A B C|$ the area of $\triangle A B C$ we have

$$
\begin{aligned}
& \int_{A B C}|\nabla \phi|^{2} d \mathbf{x}=\left(\left[\frac{t_{0}-t_{j}}{|B K|}\right]^{2}+\left[\frac{t_{i}-t_{k}}{|A C|}\right]^{2}\right)|A B C| \\
& =\frac{|A C||A B| \sin (\angle B A C)}{2}\left(\left[\frac{\left(t_{i}-t_{j}\right)+\left(t_{k}-t_{i}\right) \frac{|A B| \cos (\angle B A C)}{|A C|}}{|A B| \sin (\angle B A C)}\right]^{2}+\left[\frac{t_{i}-t_{k}}{|A C|}\right]^{2}\right) \\
& =\frac{|A C||A B| \sin (\angle B A C)}{2} \\
& \times\left(\left[\left(t_{i}-t_{j}\right) \frac{1}{|A B| \sin (\angle B A C)}+\left(t_{k}-t_{i}\right) \frac{|A B| \cos (\angle B A C)}{|A C||A B| \sin (\angle B A C)}\right]^{2}+\left[\frac{t_{i}-t_{k}}{|A C|}\right]^{2}\right) \\
& \leq|A C||A B| \sin (\angle B A C) \\
& \times\left(\left(t_{i}-t_{j}\right)^{2} \frac{1}{|A B|^{2} \sin ^{2}(\angle B A C)}+\left(t_{k}-t_{i}\right)^{2} \frac{1+\cot ^{2}(\angle B A C)}{|A C|^{2}}\right) \\
& =\frac{1}{\sin (\angle B A C)}\left(\left(t_{i}-t_{j}\right)^{2} \frac{|A C|}{|A B|}+\left(t_{k}-t_{i}\right)^{2} \frac{|A B|}{|A C|}\right) \\
& \leq \frac{2}{\sin (\angle B A C)}\left(\left(t_{i}-t_{j}\right)^{2}+\left(t_{k}-t_{i}\right)^{2}\right) .
\end{aligned}
$$

Hence

$$
\left.\int_{A B C}|\nabla \phi|^{2} d \mathbf{x} \leq \frac{2}{\sin (\angle B A C)}\left(\left(t_{i}-t_{j}\right)^{2}+\left(t_{k}-t_{i}\right)^{2}\right)\right)
$$

In terms of the parameter $\beta=\beta_{j k}^{\max }$ we have a bound

$$
\frac{1}{\sin (\angle B A C)}=\frac{1}{\sin \left(\pi / 2-\angle x_{k} x_{j} O\right)} \leq \frac{1}{\sqrt{1-\left(\beta_{j k}^{\max }\right)^{2}}} .
$$

therefore

$$
\int_{A B C}|\nabla \phi|^{2} d \mathbf{x} \leq \frac{2}{\sqrt{1-\beta^{2}}}\left(\left(t_{i}^{0}-t_{j}^{0}\right)^{2}+\left(t_{k}^{0}-t_{i}^{0}\right)^{2}\right) .
$$

By lemma 6 we have

\section{Corollary 1 Assume}

$$
\beta_{j k}^{\max }=\max \left(\beta_{i j}^{\max }, \beta_{k j}^{\max }, \beta_{k i}^{\max }\right) .
$$


If $\mathcal{N}=3$

$$
\int_{\Delta_{i j k}}|\nabla \phi|^{2} d x \leq \frac{4}{\sqrt{3}}\left(1+\frac{\delta}{2 R}\right)\left(\left(t_{i}^{0}-t_{j}^{0}\right)^{2}+\left(t_{k}^{0}-t_{i}^{0}\right)^{2}\right) .
$$

If $\mathcal{N}=4$

$$
\int_{\Delta_{i j k}}|\nabla \phi|^{2} d x \leq 2 \sqrt{2}\left(1+\frac{\delta}{2 R}\right)\left(\left(t_{i}^{0}-t_{j}^{0}\right)^{2}+\left(t_{k}^{0}-t_{i}^{0}\right)^{2}\right) .
$$

If $\mathcal{N}$ is arbitrary

$$
\int_{\Delta_{i j k}}|\nabla \phi|^{2} d x \leq \frac{\mathcal{N}}{\sqrt{1-1 / \mathcal{N}^{2}}}\left(1+\frac{\delta}{2 R}\right)\left(\left(t_{i}^{0}-t_{j}^{0}\right)^{2}+\left(t_{k}^{0}-t_{i}^{0}\right)^{2}\right) .
$$

\section{References}

[1] Ambegaokar, V., Halperin, B.I., Langer, J,S. 1971, Hopping conductivity in disordered systems. Phys.Rev.B, 4(8), pp2612-2620 (1971).

[2] Bakhvalov N. S., Panasenko G. P., Homogenization: averaging processes in periodic media. Kluwer, Dordrecht (1989).

[3] Bensoussan A., Lions J-L., Papanicolaou G., Asymptotic Analysis for Periodic Structures, North-Holland, Amsterdam, 1978.

[4] Berlyand, L., Kolpakov, A., Network approximation in the limit of small interparticle distance of the effective properties of a high contrast random dispersed composite, Arch. Ration. Mech. Anal. 159 (2001)3, pp. 179-227.

[5] Bollobás, B., Modern graph theory. Graduate Texts in Mathematics, 184. Springer-Verlag, New York, 1998.

[6] Borcea, L., Asymptotic Analysis of Quasistatic Transport in High Contrast Conductive Media. SIAM Journal on Applied Mathematics, 59:2(1999), pp.597-639.

[7] Borcea L., Berryman J.G., Papanicolaou G., Matching pursuit for imaging high contrast conductivity. Inverse Problems, 15:4(1999), pp.811-849.

[8] Borcea, L., Papanicolaou G., Network approximation for transport properties of high contrast materials. SIAM J Appl Math, 58:2(1998), pp.501-539.

[9] Borcea L., Papanicolaou, G., Low frequency electromagnetic fields in high contrast media. Surveys on Solution Methods for Inverse Problems, D. Colton, H. W. Engl, A. Louis, J. R. McLaughlin, W. Rundell editors, Springer Vienna/New York, 2000.

[10] Bruno, O., The effective conductivity of strongly heterogeneous composites. Proc. Royal Soc. London A, 433(1991), pp. 353-381. 
[11] Clerc, J.P., Giraud, G., Laugier, J.M., Luck, J.M., The electrical conductivity of binary disordered systems, percolation clusters, fractals and related models. Advances in Physics, 39:3(1990), pp. 191-309.

[12] Ekeland, I., Temam, R.,Convex Analysis and Variational Problems. North Holland, 1976.

[13] Halperin, B.I.,Remarks on percolation and transport in networks with a wide range of bond strengths, Physica D, 38(1989), pp. 179-183.

[14] Keller, J.B., Conductivity of a Medium Containing a Dense Array of Perfectly Conducting Spheres or Cylinders or Nonconducting cylinders. J. Appl. Phys., 34:4(1963), pp. 991-993.

[15] Koplik, J., Creeping flow in two-dimensional networks. J. Fluid Mech., 119(1982), pp. 219-247.

[16] Kozlov, S.M., Geometrical aspects of averaging, Russ. Math. Surveys, 44:2(1989), pp. 91-144.

[17] McPhedran, R. Transport property of cylinder pairs of the square array of cylinders, Proc. R. Soc. London. A, 408(1986), pp. 31-43.

[18] McPhedran, R., Poladian.L, and Milton, G.W. Asymptotic studies of closely spaced, highly conducting cylinders, Proc.. R. Soc. Lond. A, 415(1988), pp. 185196.

[19] McPhedran, R., Milton, G.W. Transport properties of touching cylinder pairs and of the square array of touching cylinders. Proc. R. Soc. London A, 411(1987), pp. 313-326.

[20] Schwartz, L.M., Banavar, J.R., Halperin, B.I., Biased-diffusion calculations of effective transport in inhomogeneous continuum systems, Physical review B, 40:13(1989), pp. 9155-9161.

[21] Shah, C.B., Yortsos, Y.C.,The permeability of strongly-disordered systems, Physics of Fluids, 8:1(1996), pp. 280-282.

[22] Smythe, W. R., Static and dynamic electricity. 2nd edition, McGraw-Hill, NY, Toronto, London, 1950. 